\title{
Algas marinhas bentônicas da região de Cabo Frio e arredores: SÍNTESE DO CONHECIMENTO
}

\author{
Poliana S. Brasileiro', Yocie Yoneshigue-Valentin ${ }^{2}$, Ricardo da G. Bahia', \\ Renata P. Reis ${ }^{l} \&$ Gilberto Menezes Amado Filho ${ }^{1,3}$
}

\begin{abstract}
Resumo
(Algas marinhas bentônicas da região de Cabo Frio e arredores: síntese do conhecimento) Nas últimas décadas, foram realizados diversos estudos sobre as algas marinhas bentônicas da região de Cabo Frio (RCF), entretanto essa informação está dispersa em publicações avulsas, dissertações e teses. Neste contexto, o objetivo deste trabalho é realizar a revisão da literatura sobre as algas marinhas bentônicas da RCF e fornecer uma listagem detalhada dos táxons com uma análise da composição florística e distribuição geográfica desta importante região do litoral brasileiro. Foram listados 339 táxons infragenéricos, distribuídos em 76 Chlorophyta, 60 Ochrophyta e 203 Rhodophyta. Os municípios com maior número de táxons foram os de Armação dos Búzios (212)e Arraial do Cabo (207). Ao comparar os 339 táxons encontrados com os registrados para o litoral brasileiro, 20 apresentam distribuição geográfica restrita a RCFe 8 possuem afinidade com águas frias. As espécies Pseudolithoderma moreirae Yoneshigue \& Boudouresque e Gracilaria yoneshigueana Gurgel, Fredericq \& J. Norris são endêmicas da RCF. A partir dos dados reunidos que indicam a elevada riqueza e a presença de elevado número de espécies com distribuição discontínua e restrita, podese afirmar que a RCF é uma das mais importantes áreas da diversidade de algas do Brasil.
\end{abstract}

Palavras-chave: florística, região de Cabo Frio, ressurgência, estado do Rio de Janeiro, algas marinhas bentônicas.

\section{Abstract}

(Benthic marine algae from Cabo Frio region and surroundings: synthesis of knowledge) At the last decades, several studies were done about benthic marine algae from Cabo Frio region (RCF), meanwhile the obtained information is scattered in specific publication, monographs and thesis. In this context, the aim of this work is to revise the literature about marine algae from RCF, providing a detailed list of taxa, and analyzing the floristic composition and geographical distribution of benthic marine algae of this importance region from the Brazilian coast. It was found 339 infrageneric taxa, distributed in 76 Chlorophyta, 60 Ochrophyta and 203 Rhodophyta. The municipalities with higher number of taxa were Armação dos Búzios (212) and Arraial do Cabo (207). It was found that 20 of the 339 taxa listed presented distribution restricted to RCF when comparing with the taxa registered to the Brazilian coast and that 8 taxa presented affinities with temperate waters. The species Pseudolithoderma moreirae Yoneshigue \& Boudouresque and Gracilaria yoneshigueana Gurgel, Fredericq \& J. Norris are endemic to RCF. From the obtained data that indicates an elevate species richness and the presence of number species with restricted and discontinued distribution we can affirm that RCF is one of the most important diversity center of marine algae in Brazil.

Key words: floristic, Cabo Frio region, upwelling, Rio de Janeiro State, benthic marine algae.

\section{INTRODUÇÃO}

A região de Cabo Frio (RCF), com 193 km de zona costeira, situa-se na porção central do litoral do estado do Rio de Janeiro (Fig. 1) e é considerada uma das mais importantes áreas turísticas do Brasil. Está situada entre o Município de Rio das Ostras, ao norte, e o Município de Maricá, ao sul, abrangendo aproximadamente $24 \%$ dos $850 \mathrm{~km}$ da costa do estado do Rio de Janeiro (CILSJ 2008).
A RCF apresenta um extenso complexo lagunar, característica que nomeia parte da área como Região dos Lagos. Neste complexo, destaca-se a Lagoa de Araruama com uma área de $215 \mathrm{~km}^{2}$ e que tem como particularidade a alta salinidade de suas águas (André et al. 1981; Barbiére 1985).

A RCF encontra-se sob influência do fenômeno oceanográfico da ressurgência, que é caracterizado pela substituição de águas

Artigo recebido em 05/2008. Aceito para publicação em 02/2009.

${ }^{1}$ Instituto de Pesquisas Jardim Botânico do Rio de Janeiro, R. Pacheco Leão 915, 22460-030, Rio de Janeiro, RJ, Brasil. ${ }^{2}$ Departamento de Botânica, Instituto de Biologia, Centro de Ciências da Saúde, Universidade Federal do Rio de Janeiro (UFRJ). Ilha do Fundão. Av. Brigadeiro Trompowsky, s.n., 21941-900, Rio de Janeiro, RJ, Brasil.

${ }^{3}$ Autor para correspondência: gfilho@jbrj.gov.br 
costeiras quentes por águas com baixas temperaturas $\left(\leq 18^{\circ} \mathrm{C}\right)$, ricas em nutrientes que se deslocam do fundo para a superfície, provenientes da região central do Atlântico Sul, denominada de Água Central do Atlântico Sul (ACAS). A ocorrência desse fenômeno é mais comum no período entre a primavera e o verão, sendo atribuída a dois fatores principais: o predomínio de ventos de direção nordeste e a quebra abrupta do sentido de orientação da plataforma continental (de norte-sul para leste-oeste), que favorece a ascenção de águas mais profundas (Moreira da Silva 1968, 1971; Mascarenhas \& Miranda 1971; Silva et al. 2006).

Os estudos sobre a ressurgência nesta região tiveram início na década de $50 \mathrm{com}$ o trabalho realizado por Allard (1955) e desde então diversos pesquisadores desenvolveram trabalhos na área, como Emilson (1961), Moreira da Silva (1968, 1971), Mascarenhas \& Miranda
(1971), Rodrigues (1973), Signorini (1978), Valentin (1974, 1983, 1984), Valentin et al. (1987), Palacios (1993), Torres Jr. (1995), entre outros.

Em função do fenômeno da ressurgência, a RCF tem sido apontada como área de elevada importância biogeográfica para diversos grupos de organismos marinhos, incluindo as algas bentônicas, representando o limite de distribuição geográfica de diversos táxons (Oliveira Filho 1977; Yoneshigue-Valentin \& Valentin 1992). Deste modo, nesta região podem ser encontrados táxons tipicamente tropicais, bem como, táxons típicos de regiões temperadas com afinidade por águas mais frias (Yoneshigue-Valentin \& Valentin 1992).

Nas últimas décadas foram realizados diversos estudos sobre algas marinhas bentônicas da RCF, entretanto, essa informação está dispersa em publicações avulsas, dissertações e teses. Neste contexto, esse trabalho tem

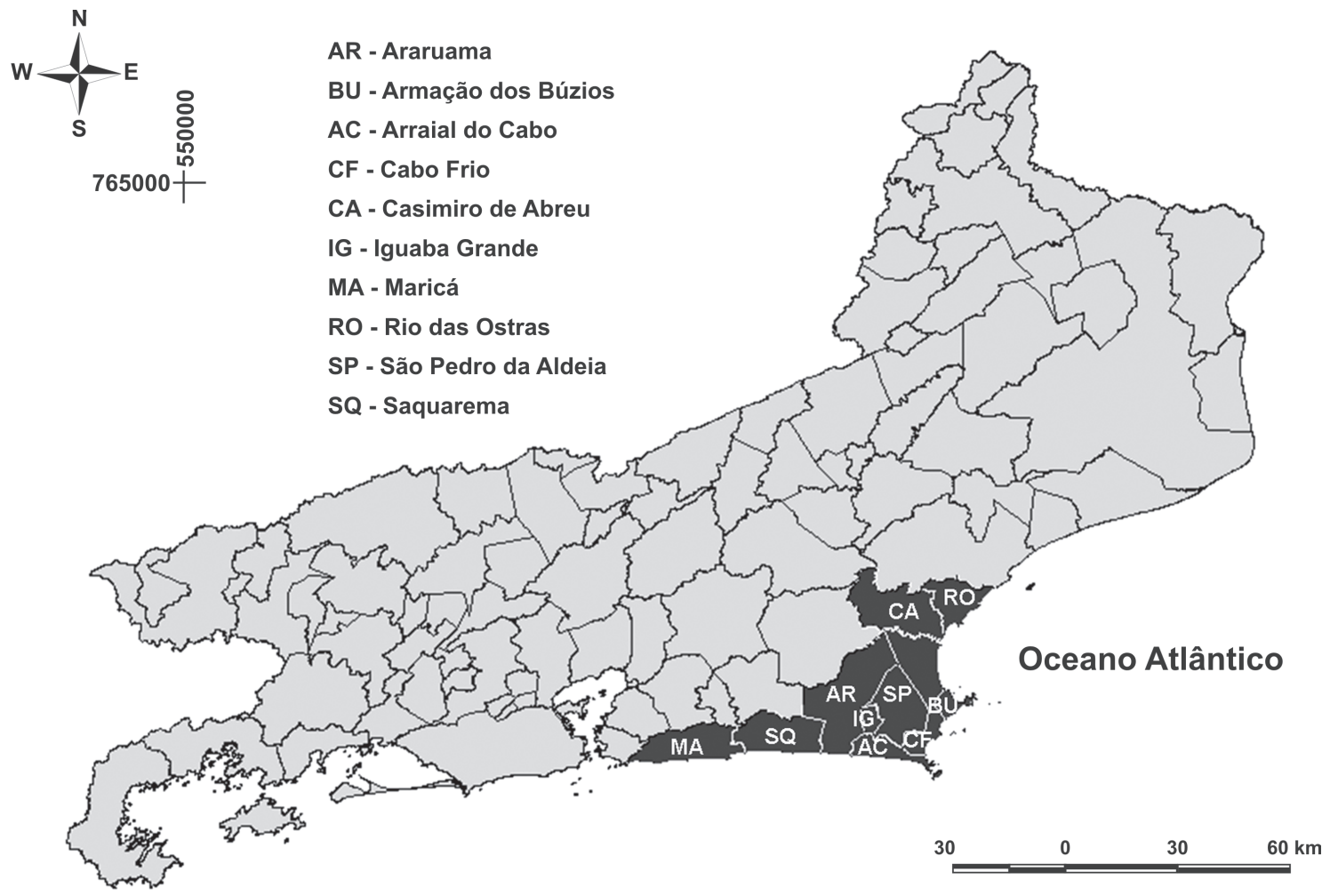

Figura 1 - Mapa do estado do Rio de Janeiro com a localização dos municípios da região de Cabo Frio e arredores analisados nesta revisão. 
como objetivo realizar a revisão da literatura sobre as algas marinhas bentônicas da RCF, fornecer uma listagem detalhada dos táxons e analisar a composição florística e a distribuição geográfica das algas marinhas bentônicas desta importante região do litoral brasileiro.

\section{Material e Métodos}

Foi realizada uma revisão da literatura, até o ano de 2008, que faz referência aos táxons infragenéricos coletados nos municípios que compõem a RCF. Esses táxons foram organizados em uma listagem, contendo informações sobre a distribuição geográfica e específica para os municípios da RCF, além das referências bibliográficas que incluem a citação para a região. Foram considerados os municípios que estão sob influência direta ou indireta do fenômeno da ressurgência, ou seja, Maricá, Saquarema, Araruama, Iguaba Grande, São Pedro da Aldeia, Cabo Frio, Arraial do Cabo, Armação dos Búzios, Casimiro de Abreu e Rio das Ostras (Fig. 1). As informações sobre a composição florística da região e suas respectivas referências bibliográficas foram reunidas com o auxílio da base de dados (Amado Filho \& Bahia 2008).

Além dos sítios ao longo da costa, foram considerados também sítios de coleta distante da costa, como os estudados por YoneshigueValentin et al. (2006), com presença de macroalgas de profundidade e cuja localização não é atribuída oficialmente a nenhum município. Nesses casos, os municípios foram determinados a partir da latitude dos sítios.

Os dados referentes à distribuição geográfica mundial dos táxons foram obtidos em Guiry \& Guiry (2008). Já os dados referentes à distribuição geográfica na costa brasileira foram obtidos em Oliveira et al. (2008). A nomenclatura e organização dos táxons nas suas respectivas ordens e famílias seguiram o proposto por Wynne (2005). Os registros de táxons que não foram encontrados em Wynne (2005) tiveram sua nomenclatuara atualizada de acordo com Guiry \& Guiry (2008).

\section{Resultados e Discussão}

Ao todo, foram analisadas 34 referências bibliográficas (Tab. 1), das quais foram identificados 83 sítios de coleta na RCF (Tab. 2). Foi encontrado um total de 339 táxons infragenéricos, distribuídos em 76 Chlorophyta, 60 Ochrophyta e 203 Rhodophyta referenciados para a RCF (Tab. 3). Entre os 83 sítios amostrados, os municípios com maior número de locais de coleta estudados foram: Armação dos Búzios (19 sítios inventariados), seguido por Arraial do Cabo e Cabo Frio (cada um com 17 sítios analisados), enquanto que Iguaba Grande e Maricá estão representados por apenas dois sítios e Casimiro de Abreu por um sítio (Tab. 2).

Os táxons estão classificados em 27 ordens, 57 famílias e 154 gêneros. As ordens mais representativas foram: Cladophorales, entre as clorófitas, com 32 táxons; Ectocarpales, entre as ocrofíceas, com 24 táxons e Ceramiales, entre as rodofíceas, com 99 táxons. Rhodomelaceae (44 táxons), Ceramiaceae (39 táxons), Cladophoraceae (19 táxons), Dictyotaceae (13 táxons) e Sargassaceae (10 táxons) foram as famílias mais representativas. Os gêneros que mais contribuíram em número de táxons foram Ceramium (11 táxons), Sargassum (10), Ulva (9), Chaetomorpha (8), Cladophora (8) e Polysiphonia (8).

Em relação ao número de táxons por município (Fig. 2), a maior riqueza foi encontrada em Armação dos Búzios (212 táxons), seguido pelos municípios de Arraial do Cabo (207 táxons) e de Cabo Frio (155). A menor riqueza foi observada nos municípios de Iguaba Grande (7 táxons), Casimiro de Abreu (13 táxons), Araruama (14) e São Pedro da Aldeia (22). Essa baixa riqueza infragenérica pode estar associada com a baixa disponibilidade de substrato consolidado, além da alta temperatura, luminosidade e salinidade presente nas porções internas da Lagoa de Araruama, onde estes municípios foram amostrados (Reis \& Yoneshigue-Valentin 1996). Em relação a Casimiro de Abreu, é provável que a baixa riqueza infragenérica detectada esteja 
Tabela 1 - Referêcias bibliografias que citam os táxons coletados nos municípios incluídos na região de Cabo Frio e arredores, estado do Rio de Janeiro (RJ).

\begin{tabular}{ll}
\hline \multicolumn{2}{c}{ Autor(es) (data da publicação) } \\
\hline 1. Amado Filho (1991) & 18. Reis-Santos (1990) \\
2. Amado Filho \& Yoneshigue-Valentin (1990/92) & 19. Reis \& Yoneshigue-Valentin (1996) \\
3. Barreto (1996) & 20. Reis \& Yoneshigue-Valentin (1998) \\
4. Barros-Barreto et al. (2006) & 21. Széchy \& Cordeiro-Marino (1991) \\
5. Bravin et al. (1999) & 22. Széchy (1996) \\
6. Bravin \& Yoneshigue-Valentin (2002) & 23. Tâmega \& Figueiredo (2005) \\
7. Cassano (1997) & 24. Teixeira et al. (1985) \\
8. Cassano et al.2004 & 25. Villaça (1988) \\
9. Guimarães et al. (1986) & 26. Yoneshigue \& Figueiredo (1983) \\
10. Guimarães \& Coutinho (1996) & 27. Yoneshigue \& Oliveira Filho (1984) \\
11. Gurgel(1997) & 28. Yoneshigue (1985) \\
12. Gurgel et al. 2004 & 29. Yoneshigue \& Villaça (1986) \\
13. Gurgelet al. (2008) & 30. Yoneshigue et al. (1986) \\
14. Mitchell et al. (1979) & 31. Yoneshigue \& Valentin (1988) \\
15. Moura (2000) & 32. Yoneshigue \& Villaça (1989) \\
16. Muniz et al. (2003) & 33. Yoneshigue-Valentin et al. (2003) \\
17. Oigman-Pszczol et al. (2004) & 34. Yoneshigue-Valentinet al. (2006)
\end{tabular}

associada à carência de estudos na área, já que até o presente, apenas o trabalho de Széchy \& Cordeiro-Marino (1991) sobre as feofíceas do norte fluminense inclui um sítio de coleta nesse município.

Apenas duas espécies, Ulva flexuosa e Cladophora vagabunda, foram citadas para todos os municípios (com exceção de Casimiro de Abreu) enquanto que 127 táxons (38\%) foram exclusivos a um dos dez municípios da RCF. Arraial do Cabo, Rio das Ostras e Armação dos Búzios são os municípios com os maiores números de táxons exclusivos (42, 33, 32 táxons, respectivamente). Os menores números de táxons exclusivos foram encontrados em Maricá e Araruama, ambos com apenas dois táxons (Cladophoropsis macromeris e Gracilaria mammillaris para Maricá; Boodleopsis pusilla e Cladophora montagneana para Araruama). Cabo Frio e Saquarema apresentaram um número intermediário de táxons exclusivos, com oito e nove táxons, respectivamente.
Quanto ao número de táxons por sítio analisado, a maior riqueza foi registrada para a Praia Rasa (Município de Armação de Búzios) com um total de 171 táxons, seguida por Ponta da Cabeça (Município de Arraial do Cabo) e Ponta do Pai Vitório (Município de Armação de Búzios), com 107 e 104 táxons, respectivamente (Fig.3). Trinta e oito sítios apresentaram número de táxons inferior a 10 (Tab. 3), o que sugere a necessidade de mais amostragens para alguns destes locais.

Ao comparar os 339 táxons coletados na RCF com os citados para todo o estado do Rio de Janeiro (Amado Filho \& Bahia 2008), observa-se que esta região apresenta $77 \%$ dos táxons coletados em todo o estado (441 táxons), e que 78 táxons são restritos a esta região. Destes 78, 16 foram coletados em profundidade (Aglaothamnion halliae, Anadyomene stellata var. floridana, Botryocladia pyriformis, Callithamniella tingitana, Caulerpa pusilla, Dasya ocellata, Microdictyon aghardianum, Microdictyon 
Tabela 2 - Sítios de coleta analisados no presente estudo, por município, com suas respectivas coordenadas geográficas e número total de táxons referenciados para cada local.

\begin{tabular}{|c|c|c|c|c|}
\hline Sítio de coleta (nome popular) & Município & $\begin{array}{l}\text { Latitude } \\
\text { (S) }\end{array}$ & $\begin{array}{l}\text { Longitude } \\
\text { (W) }\end{array}$ & $\begin{array}{l}N^{\circ} \text { de } \\
\text { táxons }\end{array}$ \\
\hline Enseada de Parati & Araruama & $22^{\circ} 52^{\prime} 14^{\prime \prime}$ & $42^{\circ} 17^{\prime} 22^{\prime \prime}$ & 5 \\
\hline Ponta das Andorinhas & Araruama & $22^{\circ} 52^{\prime} 37^{\prime \prime}$ & $42^{\circ} 15^{\prime} 31^{\prime \prime}$ & 7 \\
\hline Porto dos Leites & Araruama & $22^{\circ} 53^{\prime} 01^{\prime \prime}$ & $42^{\circ} 22^{\prime} 51^{\prime \prime}$ & 7 \\
\hline Praia de Araruama & Araruama & $22^{\circ} 52^{\prime} 38^{\prime \prime}$ & $42^{\circ} 19^{\prime} 29^{\prime \prime}$ & 8 \\
\hline Praia Seca & Araruama & $22^{\circ} 55^{\prime} 24^{\prime \prime}$ & $42^{\circ} 18^{\prime} 01^{\prime \prime}$ & 6 \\
\hline $\begin{array}{l}\text { Saco entre a Ponta das Marrecas e } \\
\text { a Ponta do Anzol }\end{array}$ & Araruama & $22^{\circ} 54^{\prime} 33^{\prime \prime}$ & $42^{\circ} 20^{\prime} 20^{\prime \prime}$ & 4 \\
\hline Trapiche dos Ingleses & Araruama & $22^{\circ} 54^{\prime} 19^{\prime \prime}$ & $42^{\circ} 22^{\prime} 43^{\prime \prime}$ & 5 \\
\hline Ilha do Caboclo & Armação dos Búzios & $22^{\circ} 45^{\prime} 06^{\prime \prime}$ & $41^{\circ} 53^{\prime} 10^{\prime \prime}$ & 2 \\
\hline Ponta da Lagoinha & Armação dos Búzios & $22^{\circ} 46^{\prime} 24^{\prime \prime}$ & $41^{\circ} 52^{\prime} 35^{\prime \prime}$ & 62 \\
\hline Ponta de João Fernandes & Armação dos Búzios & $22^{\circ} 44^{\prime} 22^{\prime \prime}$ & $41^{\circ} 52^{\prime} 43^{\prime \prime}$ & 37 \\
\hline Ponta do Mangue & Armação dos Búzios & $22^{\circ} 45^{\prime} 29^{\prime \prime}$ & $41^{\circ} 54^{\prime} 42^{\prime \prime}$ & 3 \\
\hline Ponta do Pai Vitório & Armação dos Búzios & $22^{\circ} 43^{\prime} 53^{\prime \prime}$ & $41^{\circ} 57^{\prime} 44^{\prime \prime}$ & 104 \\
\hline Praia Azeda & Armação dos Búzios & $22^{\circ} 44^{\prime} 42^{\prime \prime}$ & $41^{\circ} 52^{\prime} 52^{\prime \prime}$ & 4 \\
\hline Praia Brava & Armação dos Búzios & $22^{\circ} 45^{\prime} 13^{\prime \prime}$ & $41^{\circ} 52^{\prime} 09^{\prime \prime}$ & 11 \\
\hline Praia da Ferradura & Armação dos Búzios & $22^{\circ} 46^{\prime} 24^{\prime \prime}$ & $41^{\circ} 53^{\prime} 11^{\prime \prime}$ & 72 \\
\hline Praia da Ferradurinha & Armação dos Búzios & $22^{\circ} 46^{\prime} 49^{\prime \prime}$ & $41^{\circ} 52^{\prime} 59^{\prime \prime}$ & 49 \\
\hline Praia da Tartaruga & Armação dos Búzios & $22^{\circ} 45^{\prime} 17^{\prime \prime}$ & $41^{\circ} 54^{\prime} 15^{\prime \prime}$ & 14 \\
\hline Praia das Caravelas & Armação dos Búzios & $22^{\circ} 48^{\prime} 53^{\prime \prime}$ & $41^{\circ} 57^{\prime} 14^{\prime \prime}$ & 1 \\
\hline Praia das Focas & Armação dos Búzios & $22^{\circ} 45^{\prime} 56^{\prime \prime}$ & $41^{\circ} 52^{\prime} 23^{\prime \prime}$ & 10 \\
\hline Praia de Geribá & Armação dos Búzios & $22^{\circ} 46^{\prime} 41^{\prime \prime}$ & $41^{\circ} 54^{\prime} 17^{\prime \prime}$ & 5 \\
\hline Praia de João Fernandes & Armação dos Búzios & $22^{\circ} 44^{\prime} 33^{\prime \prime}$ & $41^{\circ} 52^{\prime} 55^{\prime \prime}$ & 1 \\
\hline Praia de João Fernandinho & Armação dos Búzios & $22^{\circ} 44^{\prime} 28^{\prime \prime}$ & $41^{\circ} 52^{\prime} 53^{\prime \prime}$ & 2 \\
\hline Praia do Canto & Armação dos Búzios & $22^{\circ} 45^{\prime} 02^{\prime \prime}$ & $41^{\circ} 53^{\prime} 44^{\prime \prime}$ & 3 \\
\hline Praia do Forno & Armação dos Búzios & $22^{\circ} 45^{\prime} 48^{\prime \prime}$ & $41^{\circ} 52^{\prime} 26^{\prime \prime}$ & 62 \\
\hline Praia dos Ossos & Armação dos Búzios & $22^{\circ} 44^{\prime} 55^{\prime \prime}$ & $41^{\circ} 52^{\prime} 54^{\prime \prime}$ & 5 \\
\hline Praia Rasa & Armação dos Búzios & $22^{\circ} 44^{\prime} 00^{\prime \prime}$ & $41^{\circ} 57^{\prime} 25^{\prime \prime}$ & 169 \\
\hline Enseada da Massambaba & Arraial do Cabo & $22^{\circ} 56^{\prime} 32^{\prime \prime}$ & $42^{\circ} 05^{\prime} 29^{\prime \prime}$ & 2 \\
\hline Enseada do Acaira & Arraial do Cabo & $22^{\circ} 56^{\prime} 29^{\prime \prime}$ & $42^{\circ} 08^{\prime} 57^{\prime \prime}$ & 7 \\
\hline Enseada dos Coroinhas & Arraial do Cabo & $22^{\circ} 55^{\prime} 36^{\prime \prime}$ & $42^{\circ} 14^{\prime} 07^{\prime \prime}$ & 5 \\
\hline Oratório & Arraial do Cabo & $23^{\circ} 00^{\prime} 08^{\prime \prime}$ & $41^{\circ} 59^{\prime} 13^{\prime \prime}$ & 66 \\
\hline Ponta da Cabeça & Arraial do Cabo & $22^{\circ} 58^{\prime} 39^{\prime \prime}$ & $42^{\circ} 02^{\prime} 03^{\prime \prime}$ & 108 \\
\hline Ponta da Fortaleza & Arraial do Cabo & $22^{\circ} 58^{\prime} 12^{\prime \prime}$ & $42^{\circ} 00^{\prime} 39^{\prime \prime}$ & 59 \\
\hline Ponta da Massambaba & Arraial do Cabo & $22^{\circ} 54^{\prime} 09^{\prime \prime}$ & $42^{\circ} 10^{\prime} 36^{\prime \prime}$ & 5 \\
\hline Ponta do Maramutá & Arraial do Cabo & $22^{\circ} 59^{\prime} 12^{\prime \prime}$ & $41^{\circ} 59^{\prime} 34^{\prime \prime}$ & 27 \\
\hline Ponta Leste & Arraial do Cabo & $22^{\circ} 58^{\prime} 47^{\prime \prime}$ & $41^{\circ} 59^{\prime} 01^{\prime \prime}$ & 48 \\
\hline Praia do Farol & Arraial do Cabo & $22^{\circ} 59^{\prime} 37^{\prime \prime}$ & $42^{\circ} 00^{\prime} 08^{\prime \prime}$ & 82 \\
\hline Praia do Forno & Arraial do Cabo & $22^{\circ} 57^{\prime} 51^{\prime \prime}$ & $42^{\circ} 00^{\prime} 40^{\prime \prime}$ & 39 \\
\hline Praia dos Anjos & Arraial do Cabo & $22^{\circ} 58^{\prime} 42^{\prime \prime}$ & $42^{\circ} 01^{\prime} 03^{\prime \prime}$ & 45 \\
\hline Prainha & Arraial do Cabo & $22^{\circ} 57^{\prime} 18^{\prime \prime}$ & $42^{\circ} 01^{\prime} 29^{\prime \prime}$ & 98 \\
\hline Racha & Arraial do Cabo & $23^{\circ} 00^{\prime} 06^{\prime \prime}$ & $42^{\circ} 00^{\prime} 40^{\prime \prime}$ & 57 \\
\hline Saco do Inglês & Arraial do Cabo & $23^{\circ} 00^{\prime} 30^{\prime \prime}$ & $42^{\circ} 00^{\prime} 26^{\prime \prime}$ & 73 \\
\hline Saia & Arraial do Cabo & $23^{\circ} 00^{\prime} 35^{\prime \prime}$ & $42^{\circ} 00^{\prime} 13^{\prime \prime}$ & 70 \\
\hline Sonar & Arraial do Cabo & $22^{\circ} 58^{\prime} 47^{\prime \prime}$ & $42^{\circ} 01^{\prime} 58^{\prime \prime}$ & 42 \\
\hline Banco de Laminaria & Cabo Frio & $22^{\circ} 30^{\prime} 00^{\prime \prime}$ & $40^{\circ} 59^{\prime} 00^{\prime \prime}$ & 1 \\
\hline Canal de Itajuru & Cabo Frio & $22^{\circ} 52^{\prime} 27^{\prime \prime}$ & $42^{\circ} 00^{\prime} 56^{\prime \prime}$ & 16 \\
\hline D4 (Revizee) & Cabo Frio & $22^{\circ} 51^{\prime} 03^{\prime \prime}$ & $41^{\circ} 09^{\prime} 07^{\prime \prime}$ & 2 \\
\hline
\end{tabular}




\begin{tabular}{|c|c|c|c|c|}
\hline Sítio de coleta (nome popular) & Município & $\begin{array}{c}\text { Latitude } \\
(\mathrm{S})\end{array}$ & $\begin{array}{c}\text { Longitude } \\
(\mathrm{W})\end{array}$ & $\begin{array}{l}\mathrm{N}^{0} \text { de } \\
\text { táxons }\end{array}$ \\
\hline Enseada Perynas & Cabo Frio & $22^{\circ} 52^{\prime} 56^{\prime \prime}$ & $42^{\circ} 04^{\prime} 31^{\prime \prime}$ & 4 \\
\hline Entrada do Canal & Cabo Frio & $22^{\circ} 52^{\prime} 50^{\prime \prime}$ & $42^{\circ} 00^{\prime} 16^{\prime \prime}$ & 62 \\
\hline Forte de São Mateus & Cabo Frio & $22^{\circ} 53^{\prime} 21^{\prime \prime}$ & $42^{\circ} 00^{\prime} 01^{\prime \prime}$ & 70 \\
\hline Ilha do Japonês & Cabo Frio & $22^{\circ} 52^{\prime} 29^{\prime \prime}$ & $42^{\circ} 00^{\prime} 17^{\prime \prime}$ & 32 \\
\hline Ilha do Vigia & Cabo Frio & $22^{\circ} 52^{\prime} 03^{\prime \prime}$ & $41^{\circ} 58^{\prime} 41^{\prime \prime}$ & 97 \\
\hline Ponta do Ambrósio & Cabo Frio & $22^{\circ} 51^{\prime} 54^{\prime \prime}$ & $42^{\circ} 02^{\prime} 43^{\prime \prime}$ & 14 \\
\hline Ponta do Costa & Cabo Frio & $22^{\circ} 52^{\prime} 06^{\prime \prime}$ & $42^{\circ} 04^{\prime} 44^{\prime \prime}$ & 15 \\
\hline Ponta dos Macacos & Cabo Frio & $22^{\circ} 52^{\prime} 15^{\prime \prime}$ & $42^{\circ} 06^{\prime} 15^{\prime \prime}$ & 8 \\
\hline Praia Brava & Cabo Frio & $22^{\circ} 53^{\prime} 04^{\prime \prime}$ & $41^{\circ} 59^{\prime} 51^{\prime \prime}$ & 2 \\
\hline Praia das Conchas & Cabo Frio & $22^{\circ} 52^{\prime} 13^{\prime \prime}$ & $41^{\circ} 58^{\prime} 48^{\prime \prime}$ & 1 \\
\hline Praia do Forte & Cabo Frio & $22^{\circ} 53^{\prime} 03^{\prime \prime}$ & $42^{\circ} 00^{\prime} 24^{\prime \prime}$ & 81 \\
\hline Praia do Mangue & Cabo Frio & $?$ & $?$ & 1 \\
\hline Praia do Peró & Cabo Frio & $22^{\circ} 51^{\prime} 55^{\prime \prime}$ & $41^{\circ} 58^{\prime} 50^{\prime \prime}$ & 4 \\
\hline Praia dos Coqueiros & Cabo Frio & $22^{\circ} 52^{\prime} 29^{\prime \prime}$ & $42^{\circ} 02^{\prime} 21^{\prime \prime}$ & 21 \\
\hline Barra de São João & Casimiro de Abreu & $22^{\circ} 35^{\prime} 53^{\prime}$, & $41^{\circ} 59^{\prime} 21^{\prime \prime}$ & 13 \\
\hline Iguaba Grande & Iguaba Grande & $22^{\circ} 50^{\prime} 26^{\prime \prime}$ & $42^{\circ} 13^{\prime} 19^{\prime \prime}$ & 3 \\
\hline Ponta das Bananeiras & Iguaba Grande & $22^{\circ} 51^{\prime} 45^{\prime \prime}$ & $42^{\circ} 14^{\prime} 03^{\prime \prime}$ & 6 \\
\hline Jaconé & Maricá & $22^{\circ} 56^{\prime} 57^{\prime \prime}$ & $42^{\circ} 40^{\prime} 53^{\prime \prime}$ & 95 \\
\hline Ponta Negra & Maricá & $22^{\circ} 57^{\prime} 39^{\prime \prime}$ & $42^{\circ} 41^{\prime} 40^{\prime \prime}$ & 64 \\
\hline 37R (Revizee) & Rio das Ostras & $22^{\circ} 22^{\prime} 08^{\prime \prime}$ & $37^{\circ} 35^{\prime} 31^{\prime \prime}$ & 2 \\
\hline CostaAzul & Rio das Ostras & $22^{\circ} 32^{\prime} 04^{\prime \prime}$ & $41^{\circ} 55^{\prime} 49^{\prime \prime}$ & 12 \\
\hline D1 (Revizee) & Rio das Ostras & $22^{\circ} 23^{\prime} 16^{\prime \prime}$ & $37^{\circ} 36^{\prime} 54^{\prime \prime}$ & 41 \\
\hline Enseada do Mar do Norte & Rio das Ostras & $22^{\circ} 31^{\prime} 12^{\prime \prime}$ & $41^{\circ} 55^{\prime} 04^{\prime \prime}$ & 39 \\
\hline Estação 7 (Costa Norte do Estado) & Rio das Ostras & $22^{\circ} 22^{\prime} 05^{\prime \prime}$ & $37^{\circ} 36^{\prime} 00^{\prime \prime}$ & 4 \\
\hline Praia das Tartarugas & Rio das Ostras & $22^{\circ} 31^{\prime} 54^{\prime \prime}$ & $41^{\circ} 57^{\prime} 20^{\prime \prime}$ & 32 \\
\hline Praia de Itapebuçu & Rio das Ostras & $22^{\circ} 28^{\prime} 35^{\prime \prime}$ & $41^{\circ} 51^{\prime} 45^{\prime \prime}$ & 42 \\
\hline Praia dos Pescadores & Rio das Ostras & $22^{\circ} 32^{\prime} 08^{\prime \prime}$ & $41^{\circ} 56^{\prime} 13^{\prime \prime}$ & 22 \\
\hline Y2 (Revizee) & Rio das Ostras & $22^{\circ} 22^{\prime} 55^{\prime \prime}$ & $37^{\circ} 35^{\prime} 16^{\prime \prime}$ & 8 \\
\hline Boqueirão & São Pedro da Aldeia & $22^{\circ} 51^{\prime} 51^{\prime \prime}$ & $42^{\circ} 06^{\prime} 18^{\prime \prime}$ & 7 \\
\hline Ponta da Farinha & São Pedro da Aldeia & $22^{\circ} 51^{\prime} 17^{\prime \prime}$ & $42^{\circ} 11^{\prime} 37^{\prime \prime}$ & 3 \\
\hline Ponta do Cardoso & São Pedro da Aldeia & $22^{\circ} 50^{\prime} 33^{\prime \prime}$ & $42^{\circ} 07^{\prime} 33^{\prime \prime}$ & 4 \\
\hline Praia Linda & São Pedro da Aldeia & $22^{\circ} 53^{\prime} 03^{\prime \prime}$ & $42^{\circ} 07^{\prime} 42^{\prime \prime}$ & 4 \\
\hline Saco do Sorita & São Pedro da Aldeia & $22^{\circ} 51^{\prime} 24^{\prime \prime}$ & $42^{\circ} 02^{\prime} 25^{\prime \prime}$ & 18 \\
\hline São Pedro da Aldeia & São Pedro da Aldeia & $22^{\circ} 50^{\prime} 34^{\prime \prime}$ & $42^{\circ} 04^{\prime} 49^{\prime \prime}$ & 8 \\
\hline Laje de Itaúna & Saquarema & $22^{\circ} 56^{\prime} 23^{\prime \prime}$ & $42^{\circ} 28^{\prime} 30^{\prime \prime}$ & 78 \\
\hline Ponta da Barra & Saquarema & $22^{\circ} 56^{\prime} 15^{\prime \prime}$ & $42^{\circ} 29^{\prime} 24^{\prime \prime}$ & 69 \\
\hline Praia da Vila & Saquarema & $22^{\circ} 56^{\prime} 47^{\prime \prime}$ & $42^{\circ} 30^{\prime} 00^{\prime \prime}$ & 91 \\
\hline
\end{tabular}

boergesenii, Microdictyon callodictyon, Microdictyon vanbossae, Osmundea lata, Petroglossum undulatum, Phyllodictyon pulcherrimum, Pseudocodium floridanum, Pterothamnion heteromorphum, Syringoderma abyssicola).

Comparativamente a outros estados litorâneos do Brasil (Oliveira et al. 2008), constata-se que a RCF apresenta elevada riqueza de táxons de algas marinhas bentônicas (339), mesmo apresentando uma extensão de litoral restrita a $24 \%$ do estado do Rio de Janeiro. Este número é inferior a apenas àqueles observados para os estados do Espírito Santo (435 táxons) e da Bahia (384). A RCF apresenta $53 \%$ do total de táxons registrados para o litoral brasileiro (643 táxons) (Horta $e t$ al. 2001; Oliveira et al. 2008), sendo que 20 
táxons são de ocorrência restrita à RCF: Antithamnion villosum, Boodlea composita, Chaetomorpha pachynema, Cheilosporum cultratum, Dasya ocellata, Elachista minutissima, Endarachne binghamiae, Gonimophyllum africanum, Gracilaria yoneshigueana, Hapalospongidion macrocarpa, Hydrolithon samoënse, Hypneocolax stellaris, Jolyna laminarioides, Kuckuckia spinosa, Microdictyon tenuius, Porphyra leucosticta, Pseudendoclonium marinum, Pseudolithoderma moreirae, Pterothamnion heteromorphum e Ralfsia bornetii. Destes, oito são característicos de clima temperado ou polar (Antithamnion villosum, Elachista minutissima, Gonimophyllum africanum, Hapalospongidion macrocarpa, Kuckuckia spinosa, Porphyra leucosticta, Pterothamnion heteromorphum, Ralfsia bornetii). Duas espécies, Pseudolithoderma moreirae e Gracilaria yoneshigueana, são endêmicas da RCF.

Horta et al. (2001), ao estudar a distribuição e a origem das macroalgas marinhas do litoral brasileiro, propõe que o litoral seja dividido em duas regiões principais ou províncias ficogeográficas: a tropical e a temperada quente. Essas regiões foram caracterizadas por apresentarem floras relativamente homogêneas e com fisionomias geográficas

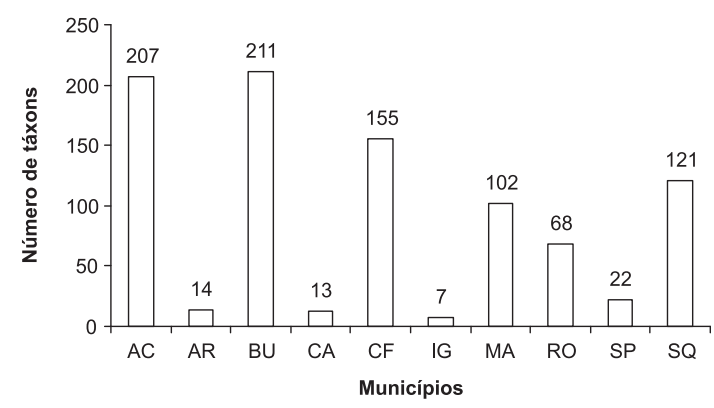

Figura 2 - Número de táxons por município analisados na Região de Cabo Frio e arredores, Estado do Rio de Janeiro. AC = Arraial do Cabo, $\mathrm{AR}=$ Araruama, $\mathrm{BU}=$ Armação dos Búzios, $\mathrm{CF}=$ Cabo Frio, $\mathrm{CA}=$ Casimiro de Abreu, IG = Iguaba Grande, MA = Maricá, $\mathrm{RO}=$ Rio das Ostras, SP = São Pedro da Aldeia e SQ = Saquarema. semelhantes. Essas duas províncias foram separadas por uma zona de transição, representada pelo estado do Espírito Santo, que apresenta grande diversidade de ambientes. A RCF também apresenta elevada diversidade que está associada, em parte, a ocorrência de espécies típicas de regiões temperadas e, como mencionado por Yoneshigue (1985) e Yoneshigue-Valentin \& Valentin (1992), é considerada uma barreira geográfica para distribuição de espécies de macroalgas, especialmente como limite norte para a ocorrência de diversos táxons. As características peculiares ocasionadas pelo fenômeno da ressurgência possibilitam o estabelecimento de táxons com maior afinidade por águas de temperatura mais amena em uma latitude tropical, que eleva a riqueza de táxons regionais.

A partir dos dados reunidos que indicam a elevada riqueza e a presença de elevado número de espécies com distribuição descontínua e restrita, pode-se afirmar que a RCF é uma das mais importantes áreas da diversidade de algas do Brasil. As informações disponibilizadas neste trabalho sobre distribuição e ocorrência das macroalgas podem ser utilizadas para a definição de áreas prioritárias para conservação através da criação ou ampliação de unidades de conservação na RCF.

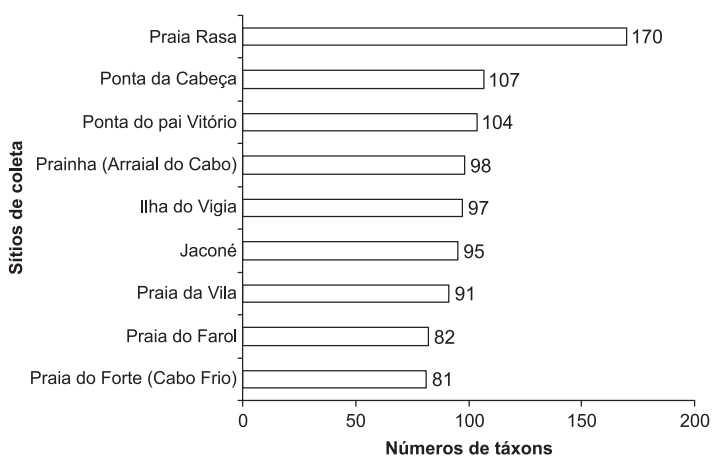

Figura 3 - Número de táxons encontrados nos sítios de coleta analisados na Região de Cabo Frio e arredores, Estado do Rio de Janeiro, que apresentaram riqueza maior que 80 táxons. 
Tabela 3 - Distribuição geográfica mundial e por municípios brasileiros, bem como referências bibliográficas (vide tabela 1) dos táxons de algas marinhas bentônicas que ocorrem na Região de Cabo Frio e arredores (RJ). $\mathrm{AC}=$ Arraial do Cabo, $\mathrm{AR}=$ Araruama, $\mathrm{BU}=\mathrm{Armação} \mathrm{dos} \mathrm{Búzios}, \mathrm{CF}=$ Cabo Frio, $\mathrm{CA}=$ Casimiro de Abreu, $\mathrm{IG}=$ Iguaba Grande, $\mathrm{MA}=$ Maricá, $\mathrm{RO}=$ Rio das Ostras, $\mathrm{SP}=$ São Pedro da Aldeia, $\mathrm{SQ}=\mathrm{Saquarema}, \mathrm{C}=$ Cosmopolita, $\operatorname{Tr}=$ Tropical, $\mathrm{Te}=$ Temperado, $\mathrm{A}=$ Oceano Atlântico, $\mathrm{A}($ Brasil) $=$ quando a citação para o Oceâno Atlântico é exclusiva para o litoral brasileiro, P = Oceano Pacífico, I = Oceano Índico, M = Mediterrâneo, MV= Mar Vermelho, ST = sub-tropical, Po = Oceano Polar.

\begin{tabular}{|c|c|c|c|}
\hline Táxons & $\begin{array}{l}\text { Distribuição } \\
\text { geográfica } \\
\text { mundial }\end{array}$ & Municípios & $\begin{array}{l}\text { Referência } \\
\text { bibliográfica }\end{array}$ \\
\hline \multicolumn{4}{|l|}{ FILOCHLOROPHYTA } \\
\hline \multicolumn{4}{|l|}{ Classe Chlorophyceae } \\
\hline \multicolumn{4}{|l|}{ TETRASPORALES } \\
\hline \multicolumn{4}{|l|}{ Palmellopsidaceae } \\
\hline Palmophyllum crassum (Naccari) Rabenh. & $\mathrm{A}, \mathrm{M}, \mathrm{P}$ & $\mathrm{RO}$ & 5,34 \\
\hline Palmophyllum umbracola W. Nelson \& Ryan & A (Brasil), $\mathrm{P}$ & $\mathrm{RO}$ & 5,34 \\
\hline Verdigellas peltata D.L. Ballant. \& J.N. Norris & $\mathrm{A}$ & $\mathrm{RO}$ & 5,34 \\
\hline \multicolumn{4}{|l|}{ Classe Ulvophyceae } \\
\hline \multicolumn{4}{|l|}{ ULVALES } \\
\hline \multicolumn{4}{|l|}{ Gayraliaceae } \\
\hline $\begin{array}{l}\text { Gayralia oxysperma (Kütz.) Vinogr. ex Scagel et al. } \\
\text { Gomontiaceae }\end{array}$ & $\mathrm{Tr}, \mathrm{Te}$ & $\mathrm{BU}, \mathrm{CF}$ & $19,18,28$ \\
\hline Blidingia minima (Nägeli ex Kütz.) Kylin & $\mathrm{C}$ & $\mathrm{CF}$ & 19,18 \\
\hline \multicolumn{4}{|l|}{ Ulvaceae } \\
\hline $\begin{array}{l}\text { Ulva chaetomorphoides (Boergesen) H.S. Hayden, } \\
\text { Blomster, Maggs, P.C. Silva, Stanhope \& Waaland }\end{array}$ & $\mathrm{A}, \mathrm{P}$ & $\mathrm{AC}, \mathrm{BU}, \mathrm{CF}$, & $19,18,28,31$ \\
\hline Ulva clathrata (Roth) C. Agardh & $\mathrm{C}$ & BU, CF, SQ & $14,19,18$ \\
\hline Ulva compressa $\mathrm{L}$. & $\mathrm{C}$ & $\mathrm{AC}, \mathrm{BU}, \mathrm{SQ}$ & $1,10,14.28,31$ \\
\hline Ulva fasciata Delile & $\mathrm{Tr}, \mathrm{Te}$ & AC, BU, CF. MA, RO, SQ & $1,10,11,14,19,20,18,22,28,31$ \\
\hline Ulva flexuosa subsp. paradoxa (C. Agardh) M.J. Wynne comb. nov. & A & AR, CF, SP, SQ & $1,19,18$ \\
\hline Ulva flexиosa Wulfen & $\mathrm{Tr}, \mathrm{Te}$ & AC, AR, CF, BU, IG MA, RO, SP, SQ & $1,10,11,14,16,19,18,22,28,31$ \\
\hline Ulva lactuca $\mathrm{L}$. & $\mathrm{C}$ & $\mathrm{AC}, \mathrm{BU}, \mathrm{CF}, \mathrm{MA}, \mathrm{RO}, \mathrm{SP}, \mathrm{SQ}$ & $1,10,11,14,16,19,20,18,22,28$ \\
\hline Ulva linza $\mathrm{L}$. & $\mathrm{C}$ & $\mathrm{AC}, \mathrm{BU}, \mathrm{MA}, \mathrm{SQ}$ & $1,10,28$ \\
\hline Ulva rigida C. Agardh & $\mathrm{C}$ & $\mathrm{AC}, \mathrm{BU}, \mathrm{CF}, \mathrm{SP}, \mathrm{SQ}$ & $1,11,19,20,18,25,28,31$ \\
\hline
\end{tabular}




\begin{tabular}{|c|c|c|c|}
\hline Táxons & $\begin{array}{c}\text { Distribuição } \\
\text { geográfica } \\
\text { mundial }\end{array}$ & Municípios & $\begin{array}{l}\text { Referência } \\
\text { bibliográfica }\end{array}$ \\
\hline \multicolumn{4}{|l|}{ Ulvellaceae } \\
\hline Entocladia viridis Reinke & $\mathrm{C}$ & $\mathrm{AC}, \mathrm{BU}, \mathrm{CF}, \mathrm{MASP}$ & $1,19,18,25,28,31$ \\
\hline Pringsheimiella scutata (Reinke) Höhn. ex Marchew. & $\mathrm{Tr}, \mathrm{Te}$ & SQ & 1 \\
\hline Pseudendoclonium marinum (Reinke) Aleem \& E. Schulz & A & $\mathrm{AC}, \mathrm{BU}, \mathrm{CF}$ & 25,28 \\
\hline Ulvella lens P. Crouan \& H. Crouan & $\mathrm{Tr}, \mathrm{Te}$ & $\mathrm{AC}$ & 25 \\
\hline \multicolumn{4}{|l|}{ PHAEOPHILALES } \\
\hline \multicolumn{4}{|l|}{ Phaeophilaceae } \\
\hline Phaeophila dendroides (P. Crouan \& H. Crouan) Batters & $\mathrm{Tr}, \mathrm{Te}$ & $\mathrm{AC}, \mathrm{AR}, \mathrm{CF}, \mathrm{SP}$ & $19,18,28,31$ \\
\hline \multicolumn{4}{|l|}{ CLADOPHORALES } \\
\hline Anadyomene linkiana D. Littler \& M. Littler & A & RO & 5,34 \\
\hline Anadyomene pavonina (J. Agardh) Wille & $\mathrm{A}, \mathrm{I}$ & $\mathrm{RO}$ & 5,34 \\
\hline Anadyomene saldanhae A.B. Joly \& E.C. Oliveira & A & RO & 34 \\
\hline Anadyomene stellata (Wulfen in Jacq.) C. Agardh & $\mathrm{Tr}, \mathrm{Te}$ & RO & 24,34 \\
\hline Anadyomene stellata var. floridana Gray & $\mathrm{Tr}, \mathrm{Te}$ & $\mathrm{RO}$ & 34 \\
\hline Microdictyon aghardianum Decne. & $\mathrm{Tr}, \mathrm{Te}$ & $\mathrm{RO}$ & 34 \\
\hline Microdictyon boergesenii Setch. & $\mathrm{A}, \mathrm{I}$ & $\mathrm{RO}$ & 34 \\
\hline Microdictyon calodictyon (Mont.) Kütz. & $\mathrm{A}, \mathrm{I}$ & $\mathrm{RO}$ & 34 \\
\hline Microdictyon tenuius J.E. Gray & $\operatorname{Tr}$ & $\mathrm{RO}$ & 34 \\
\hline Microdictyon vanbosseae Setch. & A, $\mathrm{P}$ & $\mathrm{RO}$ & 34 \\
\hline \multicolumn{4}{|l|}{ Cladophoraceae } \\
\hline Chaetomorpha aerea (Dillwyn) Kütz. & $\mathrm{Tr}, \mathrm{Te}$ & AC, BU,CF, MA, SQ & $1,11,14,16,28,31$ \\
\hline Chaetomorpha antennina (Bory) Kütz. & $\mathrm{Tr}, \mathrm{Te}$ & $\mathrm{AC}, \mathrm{BU}, \mathrm{CF}, \mathrm{MA}, \mathrm{RO}, \mathrm{SQ}$ & $1,10,14,19,18,28,31$ \\
\hline Chaetomorpha brachygona Harv. & $\mathrm{Tr}, \mathrm{Te}$ & $\mathrm{AC}, \mathrm{AR}, \mathrm{CF}, \mathrm{BU}, \mathrm{MA}, \mathrm{SP}, \mathrm{SQ}$ & $1,14,16,19,18,28,31$ \\
\hline Chaetomorpha gracilis Kütz. & $\mathrm{Tr}, \mathrm{Te}$ & $\mathrm{AC}, \mathrm{AR}, \mathrm{CF}$ & 19,18 \\
\hline Chaetomorpha linum (O.F. Müll.) Kütz. & $\mathrm{C}$ & $\mathrm{AR}, \mathrm{CF}, \mathrm{SP}$ & 19,18 \\
\hline Chaetomorpha minima Collins \& Herv. & $\mathrm{A}, \mathrm{I}$ & AR, CF, IG, SP, SQ & $14,19,18$ \\
\hline
\end{tabular}

\section{PHAEOPHILALES}

Phaeophilaceae

\section{CLADOPHORALES}

Anadyomene linkiana D. Littler \& M. Little

$\mathrm{RO}$

$\mathrm{Tr}, \mathrm{Te}$

$\mathrm{Tr}, \mathrm{T}$

Microdictyon calodictyon (Mont.) Kütz.

Microdictyon tenuius J.E. Gray

$1,11,14,16,28,31$

chyoona Harv.

$14,19,18$ 


\begin{tabular}{|c|c|c|c|}
\hline Táxons & $\begin{array}{l}\text { Distribuição } \\
\text { geográfica } \\
\text { mundial }\end{array}$ & Municípios & $\begin{array}{l}\text { Referência } \\
\text { bibliográfica }\end{array}$ \\
\hline Chaetomorpha nodosa Kütz. & A, P, Po & BU, SQ & 14 \\
\hline Chaetomorpha pachynema (Montagne) Kütz. & $\mathrm{Tr}, \mathrm{Te}$ & $\mathrm{AC}$ & 28 \\
\hline Cladophora albida (Nees) Kütz. & $\mathrm{C}$ & $\mathrm{AC}, \mathrm{SQ}$ & $1,11,28,31$ \\
\hline Cladophora brasiliana G. Martens & A & $\mathrm{AC}, \mathrm{AR}, \mathrm{CF}$ & 19,18 \\
\hline Cladophora coelothrix Kütz. & $\mathrm{C}$ & $\mathrm{AC}, \mathrm{BU}, \mathrm{MA}$, & $1,28,31$ \\
\hline Cladophora corallicola Boergesen & A & $\mathrm{AC}, \mathrm{BU}, \mathrm{SQ}$, & 1,28 \\
\hline Cladophora flexuosa (O.F. Müll.) Kütz. & $\mathrm{C}$ & $\mathrm{AC}$ & 25 \\
\hline Cladophora montagneana Kütz. & $\mathrm{Tr}, \mathrm{Te}$ & AC, AR, BU, CF, MA, IG, SP, SQ & $1,10,11,14,16,19,18,25,28,31$ \\
\hline Cladophora prolifera (Roth) Kütz. & $\mathrm{Tr}, \mathrm{Te}$ & $\mathrm{AC}, \mathrm{CF}, \mathrm{BU}, \mathrm{RO}, \mathrm{SQ}$ & $14,25,28$ \\
\hline Cladophora rupestris (L.) Kütz. & $\mathrm{C}$ & AC, CF, BU, MA, SQ & $1,10,11,19,18,25,28,31$ \\
\hline Cladophora vagabunda (L.) C. Hoek & $\mathrm{C}$ & AC, AR, BU, CF, IG, MA, RO, SP, SQ & $1,10,11,14,19,18,22,28,31$ \\
\hline Rhizoclonium africanum Kütz. & $\mathrm{Tr}, \mathrm{Te}$ & $\mathrm{AC}, \mathrm{AR}, \mathrm{CF}, \mathrm{SP}$ & 19,18 \\
\hline Rhizoclonium riparium (Roth) Kütz. ex Harv. & $\mathrm{C}$ & AR, BU, AC, CF, IG, MA, SP & $1,10,19,18,28$ \\
\hline \multicolumn{4}{|l|}{ Boodleaceae } \\
\hline Boodlea composita (Harv.) F. Brand & $\mathrm{Tr}, \mathrm{Te}$ & $\mathrm{BU}$ & 28,30 \\
\hline Phyllodictyon pulcherrimum J.E. Gray & $\operatorname{Tr}$ & $\mathrm{RO}$ & 5,34 \\
\hline \multicolumn{4}{|l|}{ Siphonocladaceae } \\
\hline Cladophoropsis macromeres W.R. Taylor & $\operatorname{Tr}$ & MA & 1 \\
\hline Cladophoropsis membranacea (C. Agardh) Boergesen & $\mathrm{Tr}, \mathrm{Te}$ & $\mathrm{AR}, \mathrm{BU}, \mathrm{CF}, \mathrm{MA}$ & $1,10,14,19,18,28$ \\
\hline \multicolumn{4}{|l|}{ Valoniaceae } \\
\hline Ernodesmis verticillata (Kütz.) Boergesen & $\operatorname{Tr}$ & CF & 18 \\
\hline Valonia macrophysa Kütz. & $\mathrm{Tr}, \mathrm{Te}$ & $\mathrm{AC}$ & 28 \\
\hline Valonia utricularis (Roth) C. Agardh & $\mathrm{Tr}, \mathrm{Te}$ & $\mathrm{RO}$ & 34 \\
\hline \multicolumn{4}{|l|}{ BRYOPSIDALES } \\
\hline \multicolumn{4}{|l|}{ Bryopsidaceae } \\
\hline Bryopsis corymbosa J. Agardh & $\mathrm{Tr}, \mathrm{Te}$ & $\mathrm{AC}, \mathrm{BU}$ & $10,28,31$ \\
\hline Bryopsis pennata J.V. Lamour. & $\mathrm{Tr}, \mathrm{Te}$ & $\mathrm{AC}, \mathrm{BU}, \mathrm{MA}, \mathrm{RO}, \mathrm{SQ}$ & $1,10,11,14,25,28,31$ \\
\hline Bryopsis plumosa (Huds.) C. Agardh & $\mathrm{C}$ & $\mathrm{AC}, \mathrm{CF}, \mathrm{BU}, \mathrm{MA}$ & $10,11,14,19,18,28,31$ \\
\hline
\end{tabular}




\begin{tabular}{|c|c|c|c|}
\hline Táxons & $\begin{array}{c}\text { Distribuição } \\
\text { geográfica } \\
\text { mundial }\end{array}$ & Municípios & $\begin{array}{c}\text { Referência } \\
\text { bibliográfica }\end{array}$ \\
\hline Derbesia marina (Lyngb.) Solier & $\mathrm{C}$ & $\mathrm{AC}, \mathrm{BU}, \mathrm{CF}, \mathrm{MA}, \mathrm{SQ}$ & $1,10,14,28,31$ \\
\hline Derbesia tenuissima (Moris \& De Not.) P. Crouan \& H. Crouan & $\mathrm{Tr}, \mathrm{Te}$ & $\mathrm{AC}, \mathrm{BU}, \mathrm{MA}, \mathrm{SQ}$ & $1,25,28$ \\
\hline Derbesia vaucheriaeformis (Harv.) J. Agardh & $\mathrm{A}, \mathrm{P}$ & $\mathrm{CF}$ & 19,18 \\
\hline \multicolumn{4}{|l|}{ Codiaceae } \\
\hline Codium decorticatum (Woodw.) M. Howe & $\mathrm{C}$ & AC, BU, CF, MA, RO, SQ & $1,14,19,18,22,28,31,34$ \\
\hline Codium intertextum Collins \& Herv. & $\operatorname{Tr}$ & AC, BU, CF, MA, RO, SQ & $1,10,14,19,18,22,25,28,31$ \\
\hline Codium isthmocladum Vickers & $\mathrm{Tr}, \mathrm{ST}$ & AC, BU, CF, MA & $14,28,31$ \\
\hline Codium spongiosum Harv. & $\mathrm{C}$ & $\mathrm{AC}, \mathrm{BU}, \mathrm{CF}$ & $14,19,18,28,31$ \\
\hline Codium taylorii P.C. Silva & $\mathrm{A}, \mathrm{P}, \mathrm{I}$ & $\mathrm{AC}, \mathrm{BU}, \mathrm{CF}, \mathrm{MA}, \mathrm{RO}, \mathrm{SQ}$ & $1,14,19,18,28$ \\
\hline \multicolumn{4}{|l|}{ Caulerpaceae } \\
\hline Caulerpa fastigiata Mont. & $\mathrm{Tr}, \mathrm{Te}$ & $\mathrm{BU}, \mathrm{CF}, \mathrm{MA}$ & $1,10,11,14,19,18,28,31$ \\
\hline Caulerpa mexicana Sond. ex Kütz. & $\operatorname{Tr}, \mathrm{Te}$ & BU, CF, RO & 14,28 \\
\hline Caulerpa pusilla (Kütz.) J. Agardh & A & $\mathrm{RO}$ & 34 \\
\hline Caulerpa racemosa (Forsskal) J. Agardh & $\mathrm{Tr}, \mathrm{Te}$ & $\mathrm{AC}, \mathrm{BU}, \mathrm{CF}$ & $10,11,28,31$ \\
\hline Caulerpa racemosa var. peltata (J.V. Lamour.) Eubank & $\mathrm{Tr}, \mathrm{Te}$ & $\mathrm{AC}, \mathrm{BU}, \mathrm{CF}, \mathrm{RO}$ & $14,19,18$ \\
\hline \multicolumn{4}{|l|}{ Halimedaceae } \\
\hline Halimeda gracilis Harv. ex J. Agardh & $\operatorname{Tr}$ & $\mathrm{RO}$ & 34 \\
\hline \multicolumn{4}{|l|}{ Pseudocodiaceae } \\
\hline Pseudocodium floridanum Dawes \& A.C. Mathieson & $\mathrm{A}, \mathrm{P}$ & $\mathrm{RO}$ & 5,34 \\
\hline \multicolumn{4}{|l|}{ Udoteaceae } \\
\hline $\begin{array}{l}\text { Boodleopsis pusilla (Collins) W.R. Taylor, } \\
\text { A.B. Joly \& Bernat. }\end{array}$ & $\mathrm{A}, \mathrm{I}, \mathrm{P}$ & AR & 19,18 \\
\hline \multicolumn{4}{|l|}{ DASYCLADALES } \\
\hline \multicolumn{4}{|l|}{ Polyphysaceae } \\
\hline Acetabularia calyculus J.V. Lamour. in Quoy \& Gaimard & $\mathrm{Tr}, \mathrm{Te}$ & AR, CF, IG, SP & $14,19,18$ \\
\hline
\end{tabular}

Tr, ST

Codium taylorii P.C. Silva

$\mathrm{AC}, \mathrm{BU}, \mathrm{CF}$

$14,19,18,28,31$

Caulerpaceae

Caulerpa mexicana Sond. ex Kütz.

Caulerpa pusilla (Kütz.) J. Agardh

Caulerpa racemosa (Forsskal) J. Agardh

(J.V.Lamour.) Eubank 


\begin{tabular}{|c|c|c|c|}
\hline Táxons & $\begin{array}{c}\text { Distribuição } \\
\text { geográfica } \\
\text { mundial }\end{array}$ & Municípios & $\begin{array}{l}\text { Referência } \\
\text { bibliográfica }\end{array}$ \\
\hline Acetabularia schenckii K. Möbius & $\mathrm{A}$ & CF, SP & 19,18 \\
\hline \multicolumn{4}{|l|}{ FILOOCHROPHYTA } \\
\hline \multicolumn{4}{|l|}{ Classe Phaeophyceae } \\
\hline \multicolumn{4}{|l|}{ DICTYOTALES } \\
\hline \multicolumn{4}{|l|}{ Dictyotaceae } \\
\hline Dictyopteris delicatula J.V. Lamour. & $\mathrm{Te}, \mathrm{Tr}$ & $\mathrm{BU}, \mathrm{CF}, \mathrm{CA}, \mathrm{MA}, \mathrm{RO}$ & $1,10,11,20,21,22,28,31,34$ \\
\hline Dictyopteris jamaicensis W.R. Taylor & $\mathrm{A}, \mathrm{P}$ & $\mathrm{RO}$ & 34 \\
\hline Dictyopteris plagiogramma (Mont.) Vickers & $\mathrm{Te}, \mathrm{Tr}$ & $\mathrm{BU}, \mathrm{CF}, \mathrm{RO}$ & $11,21,28,34$ \\
\hline Dictyota cervicornis Kütz. & $\mathrm{Te}, \mathrm{Tr}$ & AC, BU, CF, MA, RO & $\begin{array}{l}1,10,11,16,19,20,18,21,22,28 \\
30,31\end{array}$ \\
\hline Dictyota ciliolata Sond. ex Kütz. & $\mathrm{Te}, \mathrm{Tr}$ & $\mathrm{AC}, \mathrm{BU}, \mathrm{CF}, \mathrm{CA}, \mathrm{MA}, \mathrm{RO}$ & $1,10,11,21,28,31$ \\
\hline Dictyota menstrualis (Hoyt) Schnetter, Hörnig \& Weber-Peukert & $\mathrm{A}$ & $\mathrm{AC}, \mathrm{BU}, \mathrm{CF}, \mathrm{RO}$ & $10,21,25,28,31$ \\
\hline Dictyota mertensii (Mart.) Kütz. & $\mathrm{A}, \mathrm{I}, \mathrm{P}$ & BU & 28 \\
\hline Lobophora variegata (J.V. Lamour) Womersley exE. C. Oliveira & $\mathrm{Te}, \mathrm{Tr}$ & $\mathrm{AC}, \mathrm{BU}, \mathrm{RO}$ & $10,21,22,24,25,28,31,34$ \\
\hline Padina antillarum (Kütz.) Picc. & $\mathrm{Te}, \mathrm{Tr}$ & MA, SQ & 1 \\
\hline Padina boergesenii Allender \& Kraft & $\mathrm{Te}, \mathrm{Tr}$ & $\mathrm{BU}$ & 11 \\
\hline Padina gymnospora (Kütz.) Sond. & $\mathrm{Te}, \mathrm{Tr}$ & $\mathrm{AC}, \mathrm{BU}, \mathrm{CF}, \mathrm{CA}, \mathrm{RO}$ & $10,11,16,19,20,18,21,28,31$ \\
\hline Spatoglossum schroederi (C. Agardh) Kütz. & $\mathrm{A}, \mathrm{I}, \mathrm{P}$ & $\mathrm{BU}, \mathrm{RO}$ & 21,28 \\
\hline Stypopodium zonale (J.V. Lamour.) Papenf. & $\mathrm{Te}, \mathrm{Tr}$ & $\mathrm{BU}, \mathrm{RO}$ & 24,28 \\
\hline Zonaria tournefortii (J.V. Lamour.) Mont. & $\mathrm{Te}, \mathrm{Tr}$ & $\mathrm{BU}, \mathrm{RO}$ & 21,28 \\
\hline \multicolumn{4}{|l|}{ SPHACELARIALES } \\
\hline \multicolumn{4}{|l|}{ Sphacelariaceae } \\
\hline Sphacelaria brachygona Mont. & A, I, M & $\mathrm{AC}, \mathrm{BU}, \mathrm{CF}, \mathrm{MA}, \mathrm{RO}, \mathrm{SQ}$ & $1,10,16,19,18,21,28,31$ \\
\hline Sphacelaria novae-hollandiae Sond. & $\mathrm{Te}, \mathrm{Tr}$ & $\mathrm{AC}, \mathrm{BU}$ & $10,28,31$ \\
\hline Sphacelaria rigidula Kütz. & $\mathrm{C}$ & $\mathrm{AC}$ & $16,25,28,31$ \\
\hline Sphacelaria tribuloides Menegh. & $\mathrm{Te}, \mathrm{Tr}$ & $\mathrm{AC}, \mathrm{BU}, \mathrm{CF}, \mathrm{RO}, \mathrm{SQ}$ & $1,10,19,18,21,28,31$ \\
\hline \multicolumn{4}{|l|}{ SYRINGODERMATALES } \\
\hline \multicolumn{4}{|l|}{ Syringodermataceae } \\
\hline Syringoderma abyssicola (Setchell \& N.L. Gardner) Levring & $\mathrm{Te}$ & $\mathrm{CF}$ & 34 \\
\hline
\end{tabular}

\section{FILOOCHROPHYTA}

Classe Phaeophyceae

Dictyopteris delicatula J.V. Lamour.

$10,11,16,19,20,18,21,22,28$

$1,10,11,21,28,31$

$10,21,25,28,31$

$10,21,22,24,25,28,31,34$

$10,11,16,19,20,18,21,28,31$

21,28

24,28

Stypopodium zonale (J.V. Lamour.) Papenf. 


\begin{tabular}{|c|c|c|c|}
\hline Táxons & $\begin{array}{l}\text { Distribuição } \\
\text { geográfica } \\
\text { mundial }\end{array}$ & Municípios & $\begin{array}{l}\text { Referência } \\
\text { bibliográfica }\end{array}$ \\
\hline \multicolumn{4}{|l|}{ ECTOCARPALES } \\
\hline \multicolumn{4}{|l|}{ Acinetosporaceae } \\
\hline Acinetospora crinita (Carmich. ex Harv. in Hook.) Kornmann & $\mathrm{Te}, \mathrm{Tr}$ & $\mathrm{AC}, \mathrm{CA}$ & $21,25,26,28,31$ \\
\hline Feldmannia indica (Sond.) Womersley \& A. Bailey & $\mathrm{Te}, \mathrm{Tr}$ & BU & 7 \\
\hline Feldmannia irregularis (Kütz.) Hamel & $\mathrm{Te}, \mathrm{Tr}$ & $\mathrm{AC}, \mathrm{BU}, \mathrm{CF}, \mathrm{CA}, \mathrm{MA}, \mathrm{RO}, \mathrm{SQ}$ & $1,7,10,16,19,18,21,22,28,31$ \\
\hline Feldmannia simplex (H. Crouan \& P. Crouan) Hamel & $\mathrm{Te}, \mathrm{M}$ & $\mathrm{BU}, \mathrm{RO}$ & 22 \\
\hline Hincksia mitchelliae (Harv.) P.C. Silva & $\mathrm{Te}, \mathrm{Tr}$ & AC, BU, CF, CA, MA, RO, SQ & $\begin{array}{l}1,7,10,11,16,19,18,21,22,25 \\
28,31\end{array}$ \\
\hline \multicolumn{4}{|l|}{ Chordariaceae } \\
\hline Elachista minutissima W.R. Taylor & $\mathrm{Te}$ & SQ & 1,2 \\
\hline Elachistiella leptonematoides Cassano, Yonesh. \& M.J. Wynne & A (Brasil) & $\mathrm{AC}, \mathrm{CF}, \mathrm{MA}$ & $1,8,19,18,25,28,31$ \\
\hline Hecatonema floridanum (W.R. Taylor) W.R. Taylor & A & SQ & 1,2 \\
\hline Hecatonema terminale (Kütz.) Sauv. & $\mathrm{C}$ & $\mathrm{AC}$ & 25,28 \\
\hline Levringia brasiliensis (Mont.) A.B. Joly & A & AC, BU, CF, CA, MA, RO, SQ & $1,10,11,19,20,18,21,22,28,31$ \\
\hline Myrionema strangulans Grev. & $\mathrm{C}$ & SQ & 1,2 \\
\hline Nemacystus howei (W.R. Taylor) Kylin & A & $\mathrm{AC}$ & 16 \\
\hline Protectocarpus speciosus (Boergesen) Kuck. & $\mathrm{Te}, \mathrm{Tr}$ & $\mathrm{AC}, \mathrm{SQ}$ & $1,10,25,26,28,31$ \\
\hline \multicolumn{4}{|l|}{ Ectocarpaceae } \\
\hline Bachelotia antillarum (Grunow) Gerloff & $\mathrm{C}$ & $\mathrm{AC}, \mathrm{BU}, \mathrm{CF}, \mathrm{CA}, \mathrm{MA}, \mathrm{RO}, \mathrm{SQ}$ & $1,10,16,19,18,21,22,28,31$ \\
\hline Ectocarpus fasciculatus Harv. & $\mathrm{C}$ & $\mathrm{AC}$ & $25,26,28,31$ \\
\hline Ectocarpus fasciculatus var. pygmaeus (Aresch. in Kjellm.) Batters & & $\mathrm{BU}, \mathrm{CF}$ & 26,28 \\
\hline Ectocarpus rallsiae Vickers & A & $\mathrm{AC}, \mathrm{BU}, \mathrm{CF}, \mathrm{SQ}$ & $1,25,26,28,31$ \\
\hline Kuckuckia spinosa (Kütz.) Kuck. & $\mathrm{Te}$ & $\mathrm{AC}$ & $26,25,28$ \\
\hline \multicolumn{4}{|l|}{ Scytosiphonaceae } \\
\hline Chnoospora minima (K. Hering) Papenf. & $\mathrm{Te}, \mathrm{Tr}$ & $\mathrm{AC}, \mathrm{BU}, \mathrm{CF}, \mathrm{CA}, \mathrm{MA}, \mathrm{RO}, \mathrm{SQ}$ & $1,10,21,28$ \\
\hline Colpomenia sinuosa (Roth) Derbès \& Solier & $\mathrm{C}$ & AC, BU, CF, CA, MA, RO, SQ & $\begin{array}{l}1,10,11,16,19,20,18,21,22,25 \\
28,31\end{array}$ \\
\hline Endarachne binghamiae J. Agardh & $\mathrm{A}, \mathrm{I}, \mathrm{P}$ & $\mathrm{AC}, \mathrm{SQ}$ & $1,10,28$ \\
\hline Jolyna laminarioides S.M. Guim. in Guimarães et al. & $\mathrm{A}, \mathrm{I}$ & $\mathrm{BU}$ & 9 \\
\hline
\end{tabular}

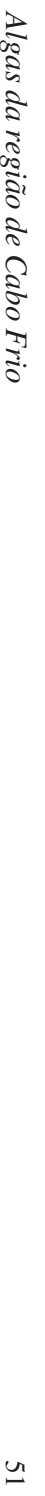




\begin{tabular}{|c|c|c|c|}
\hline Táxons & $\begin{array}{l}\text { Distribuição } \\
\text { geográfica } \\
\text { mundial }\end{array}$ & Municípios & $\begin{array}{l}\text { Referência } \\
\text { bibliográfica }\end{array}$ \\
\hline Petalonia fascia (O.F. Müll.) Kuntze & $\mathrm{C}$ & $\mathrm{AC}, \mathrm{BU}, \mathrm{MA}, \mathrm{RO}, \mathrm{SQ}$ & $1,10,21,28$ \\
\hline Rosenvingea sanctae-crucis Boergesen & $\mathrm{A}, \mathrm{I}$ & $\mathrm{AC}$ & 28 \\
\hline \multicolumn{4}{|l|}{ LAMINARIALES } \\
\hline \multicolumn{4}{|l|}{ Laminariaceae } \\
\hline Laminaria abyssalis A.B. Joly \& E.C. Oliveira & A (Brasil) & $\mathrm{CF}, \mathrm{RO}$ & 34 \\
\hline \multicolumn{4}{|l|}{ FUCALES } \\
\hline \multicolumn{4}{|l|}{ Sargassaceae } \\
\hline Sargassum cymosum C. Agardh & $\mathrm{A}, \mathrm{I}$ & $\mathrm{CF}, \mathrm{RO}$ & $11,21,28$ \\
\hline Sargassum cymosum var. nanum E. de Paula \& E.C. Oliveira & A (Brasil) & $\mathrm{BU}, \mathrm{CF}, \mathrm{CA}, \mathrm{MA}, \mathrm{RO}, \mathrm{SQ}$ & $1,21,28$ \\
\hline Sargassum filipendula C. Agardh & $\mathrm{A}, \mathrm{I}, \mathrm{P}$ & $\mathrm{BU}, \mathrm{RO}, \mathrm{SQ}$ & $1,21,28$ \\
\hline Sargassum filipendula var. montagnei (Bailey in Harv.) Grunow & A & SQ & 1 \\
\hline Sargassum filipendula var. pinnatum Grunow & A & $\mathrm{BU}$ & 28 \\
\hline Sargassum furcatum Kütz. & $\mathrm{A}, \mathrm{I}, \mathrm{M}$ & $\mathrm{AC}, \mathrm{BU}, \mathrm{CF}, \mathrm{MA}, \mathrm{SQ}$ & $1,10,11,19,18,25,28,31$ \\
\hline Sargassum stenophyllum Mart. & $\mathrm{A}, \mathrm{P}$ & BU, SQ & 1,28 \\
\hline Sargassum vulgare C. Agardh & $\mathrm{Te}, \mathrm{Tr}$ & $\mathrm{BU}, \mathrm{CF}, \mathrm{RO}, \mathrm{SQ}$ & $1,11,20,21,22,28$ \\
\hline Sargassum vulgare var. foliosissimum (J.V. Lamour) C. Agardh & A & $\mathrm{RO}, \mathrm{SQ}$ & 1,21 \\
\hline Sargassum vulgare var. nanum E. de Paula & A (Brasil) & $\mathrm{RO}$ & 22 \\
\hline \multicolumn{4}{|l|}{ Táxon de posicão incerta } \\
\hline Asteronema breviarticulatum (J. Agardh) Ouriques \& Bouzon & $\mathrm{Te}, \mathrm{Tr}$ & $\mathrm{AC}, \mathrm{BU}, \mathrm{CF}, \mathrm{CA}, \mathrm{MA}, \mathrm{RO}, \mathrm{SQ}$ & $1,7,10,21,28$ \\
\hline Asteronema rhodochortonoides (Boergesen) D.G Müller \& E.R. Parodi & $\mathrm{A}, \mathrm{I}, \mathrm{P}$ & $\mathrm{AC}, \mathrm{CF}, \mathrm{RO}, \mathrm{SQ}$ & $1,18,25$ \\
\hline \multicolumn{4}{|l|}{ RALFSIALES } \\
\hline \multicolumn{4}{|l|}{ Ralfsiaceae } \\
\hline $\begin{array}{l}\text { Hapalospongidion macrocarpa (Feldmann) Leon-Alvarez \& } \\
\text { Gonzalez-Gonzalez }\end{array}$ & $\mathrm{A}, \mathrm{M}$ & BU & 28 \\
\hline Pseudolithoderma moreirae Yonesh. \& Boudour. & A (Brasil) & $\mathrm{BU}$ & 28 \\
\hline Ralfsia bornetii Kuck. & $\mathrm{A}, \mathrm{P}$ & $\mathrm{AC}$ & 28 \\
\hline Ralfsia expansa (J. Agardh) J. Agardh & $\mathrm{A}, \mathrm{I}, \mathrm{P}$ & AC, CF, CA, BU, MA, RO, SQ & $1,10,18,21,28,31$ \\
\hline
\end{tabular}




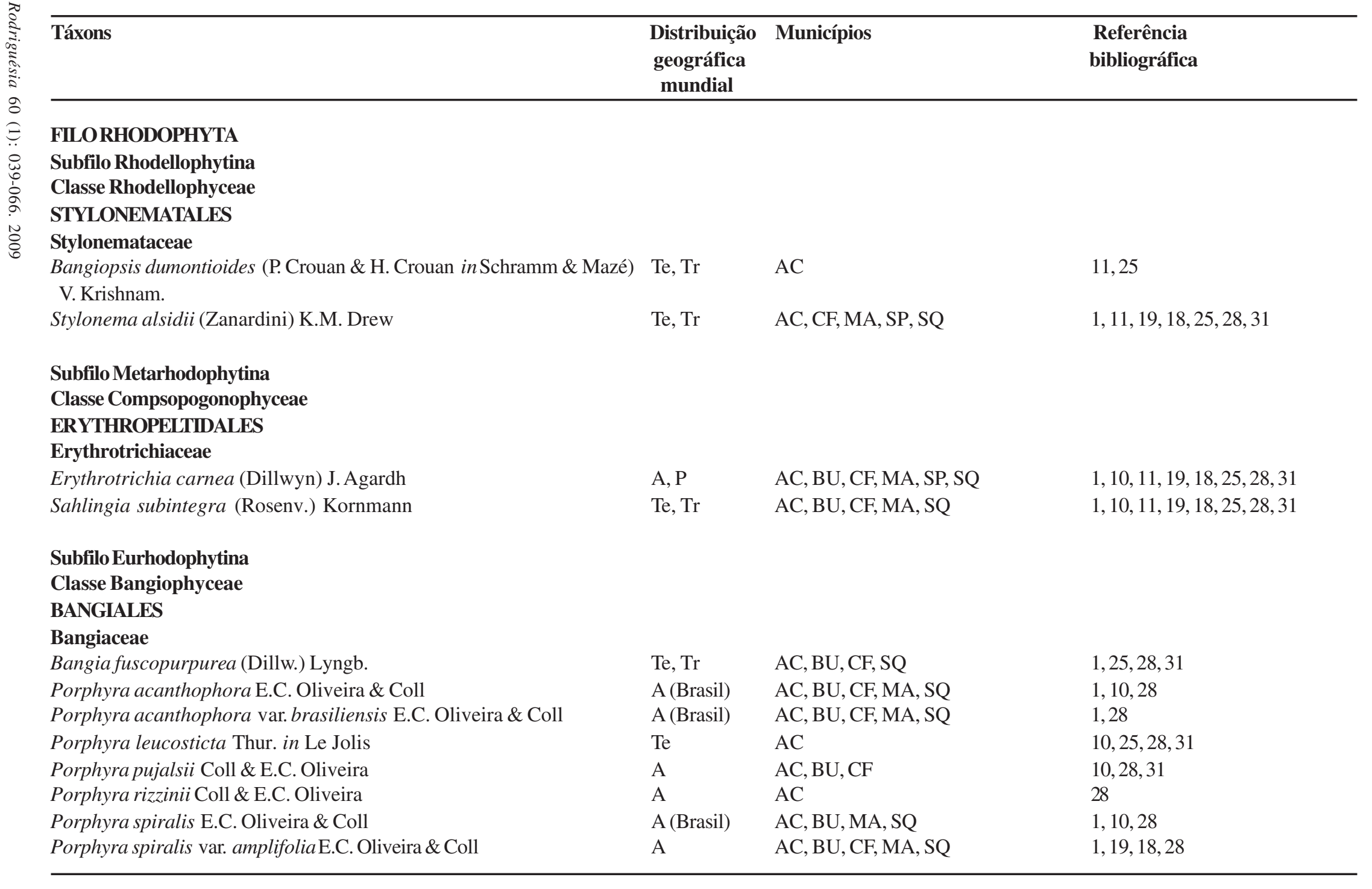

Bangiopsis dumontioides (P. Crouan \& H. Crouan in Schramm \& Mazé) Te, Tr V. Krishnam.

Subfilo Metarhodophytina

Classe Compsopogonophyceae

Erythrotrichia carnea (Dillwyn) J. Agardh

A, P AC, BU, CF, MA, SP, SQ

Subfilo Eurhodophytina

(A)

Bangia fuscopurpurea (Dillw.) Lyng .

Porphyra acanthophora var brasiliensis E.C. Oliveira \& Coll 


\begin{tabular}{|c|c|c|c|}
\hline Táxons & $\begin{array}{l}\text { Distribuição } \\
\text { geográfica } \\
\text { mundial }\end{array}$ & Municípios & $\begin{array}{l}\text { Referência } \\
\text { bibliográfica }\end{array}$ \\
\hline \multicolumn{4}{|l|}{ Classe Florideophyceae } \\
\hline \multicolumn{4}{|l|}{ Subclasse Hildenbrandiophyceae } \\
\hline \multicolumn{4}{|l|}{ HILDENBRANDIALES } \\
\hline \multicolumn{4}{|l|}{ Hildenbrandiaceae } \\
\hline Hildenbrandia rubra (Sommerf.) Menegh. & $\mathrm{C}$ & $\mathrm{AC}, \mathrm{BU}, \mathrm{CF}, \mathrm{MA}, \mathrm{SQ}$ & $1,19,18,28,31$ \\
\hline \multicolumn{4}{|l|}{ Subclasse Nemaliophycidae } \\
\hline \multicolumn{4}{|l|}{ ACROCHAETIALES } \\
\hline \multicolumn{4}{|l|}{ Acrochaetiaceae } \\
\hline Acrochaetium densum (K.M. Drew) Papenf. & $\mathrm{A}, \mathrm{P}$ & CF & 31 \\
\hline Acrochaetium flexиosum Vickers & $\mathrm{A}, \mathrm{I}$ & AC, BU, CF, MA, SQ & $1,28,31$ \\
\hline Acrochaetium globosum Boergesen & A & $\mathrm{AC}, \mathrm{BU}, \mathrm{MA}, \mathrm{SQ}$ & $1,10,28$ \\
\hline Acrochaetium hallandicum (Kylin) Hamel & A & $\mathrm{BU}$ & 28 \\
\hline Acrochaetium microscopicum (Nägeli ex Kütz.) Nägeli & $\mathrm{A}, \mathrm{I}, \mathrm{M}$ & $\mathrm{AC}, \mathrm{BU}, \mathrm{CF}, \mathrm{MA}, \mathrm{SQ}$ & $1,10,11,19,18,25,28,31$ \\
\hline \multicolumn{4}{|l|}{ CORALLINALES } \\
\hline \multicolumn{4}{|l|}{ Hapalidiaceae } \\
\hline Melobesia membranacea (Esper) J.V. Lamour. & $\mathrm{C}$ & SQ & 1 \\
\hline \multicolumn{4}{|l|}{ Corallinaceae } \\
\hline \multicolumn{4}{|l|}{ Subfamília Mastophoroideae } \\
\hline Hydrolithon samoënse (Foslie) Keats \& Chamberlain & $\mathrm{A}, \mathrm{I}, \mathrm{P}$ & BU & 23 \\
\hline Pneophyllum fragile Kütz. & $\mathrm{Tr}, \mathrm{ST}$ & $\mathrm{AC}, \mathrm{BU}, \mathrm{CF}, \mathrm{SQ}$ & $1,22,25,28,31$ \\
\hline \multicolumn{4}{|l|}{ Subfamília Corallinoideae } \\
\hline Arthrocardia flabellata (Kütz.) Manza & $\mathrm{A}, \mathrm{I}$ & $\mathrm{AC}, \mathrm{BU}, \mathrm{CF}, \mathrm{MA}, \mathrm{RO}, \mathrm{SQ}$ & $1,10,15,19,18,22,25,28,31$ \\
\hline Cheilosporum cultratum (Harv.) Aresch. & A, I, P & $\mathrm{AC}, \mathrm{BU}$ & 15 \\
\hline Cheilosporum sagittatum (J.V. Lamour.) Aresch. & A (Brasil), I & $\mathrm{AC}, \mathrm{BU}, \mathrm{CF}, \mathrm{RO}, \mathrm{SQ}$ & $10,15,22,25,28,31$ \\
\hline Corallina officinalis $\mathrm{L}$. & $\mathrm{C}$ & $\mathrm{AC}, \mathrm{BU}, \mathrm{CF}, \mathrm{MA}, \mathrm{RO}, \mathrm{SQ}$ & $1,10,11,15,19,18,25,28,31$ \\
\hline Corallina panizzoi Schnetter \& U. Richt. & A & $\mathrm{BU}, \mathrm{RO}$ & $11,15,22,28$ \\
\hline Haliptilon cubense (Mont. ex Kütz.) Gabary \& H.W. Johans. & $\operatorname{Tr}$ & $\mathrm{BU}$ & 28 \\
\hline
\end{tabular}

Classe Florideophyceae

Subclasse Nemaliophycidae

ACROCHAETIALES

Acrochaetium flexuosum Vickers

Acrochaetium globosum Boergesen

Acrochaetium hallandicum (Kylin) Hame

Hapalidiaceae

Corallinacea

$\approx$ Hydrolithon samoënse (Foslie) Keats \& Chamberlain

A, I, P

$1,22,25,28,3$ 


\begin{tabular}{|c|c|c|c|}
\hline Táxons & $\begin{array}{l}\text { Distribuição } \\
\text { geográfica } \\
\text { mundial }\end{array}$ & Municípios & $\begin{array}{l}\text { Referência } \\
\text { bibliográfica }\end{array}$ \\
\hline Jania adhaerens J.V. Lamour. & $\mathrm{Tr}, \mathrm{Te}$ & $\mathrm{AC}, \mathrm{BU}, \mathrm{CF}, \mathrm{MA}, \mathrm{RO}, \mathrm{SQ}$ & $\begin{array}{l}1,10,11,15,16,17,19,18,22, \\
25,28,31,34\end{array}$ \\
\hline Jania crassa J.V. Lamour. & $\mathrm{Tr}, \mathrm{Te}$ & $\mathrm{AC}, \mathrm{BU}, \mathrm{CF}, \mathrm{RO}$ & 15,28 \\
\hline Jania prolifera A.B. Joly & A (Brasil) & $\mathrm{RO}$ & 11,22 \\
\hline Jania rubens(L.) J.V. Lamour. & $\mathrm{Tr}, \mathrm{Te}$ & $\mathrm{AC}, \mathrm{BU}, \mathrm{CF}, \mathrm{MA}, \mathrm{SQ}$ & $1,10,19,18,28$ \\
\hline Jania ungulata f. brevior (Yendo) Yendo & A (Brasil), I, P & $\mathrm{BU}$ & 15 \\
\hline \multicolumn{4}{|l|}{ Subfamília Lithophylloideae } \\
\hline Amphiroa anastomosans Weber Bosse & $\mathrm{A}, \mathrm{I}, \mathrm{P}$ & BU & 15 \\
\hline Amphiroa beauvoisii J.V. Lamour. & $\mathrm{Tr}, \mathrm{Te}$ & AC, BU, CF, MA, RO, SQ & $1,10,11,15,17,22,25,28,31$ \\
\hline Amphiroa fragilissima (L.) J.V. Lamour. & $\mathrm{Tr}, \mathrm{Te}$ & $\mathrm{AC}, \mathrm{BU}, \mathrm{CF}, \mathrm{MA}, \mathrm{RO}, \mathrm{SQ}$ & $1,10,11,15,19,18,28,31$ \\
\hline \multicolumn{4}{|l|}{ NEMALIALES } \\
\hline Liagora ceranoides J.V. Lamour. & $\operatorname{Tr}$ & $\mathrm{CF}$ & 28 \\
\hline \multicolumn{4}{|l|}{ Galaxauraceae } \\
\hline Dichotomaria marginata (J. Ellis \& Sol.) Lamarck & $\mathrm{A}, \mathrm{T}, \mathrm{P}$ & $\mathrm{CF}$ & 28 \\
\hline Tricleocarpa fragilis(L.) Huisman \& Towns. & $\mathrm{Tr}, \mathrm{ST}$ & $\mathrm{BU}$ & 28 \\
\hline $\begin{array}{l}\text { PALMARIALES } \\
\text { Rhodothamniellaceae } \\
\text { Rhodothamniella codicola (Boergesen) Bidoux \& F. Magne }\end{array}$ & A,I & $\mathrm{AC}, \mathrm{BU}$ & 28,31 \\
\hline $\begin{array}{l}\text { Subclasse Rhodymeniophycidae } \\
\text { BONNEMAISONIALES } \\
\text { Bonnemaisoniaceae }\end{array}$ & & & \\
\hline Asparagopsis taxiformis (Delile) Trevis. & $\mathrm{Tr}, \mathrm{Te}$ & $\mathrm{AC}, \mathrm{BU}, \mathrm{CF}, \mathrm{RO}$ & $11,22,25,28,31$ \\
\hline \multicolumn{4}{|l|}{ CERAMIALES } \\
\hline $\begin{array}{l}\text { Aglaothamnion boergesenii (N. Aponte \& D.L. Ballant.) } \\
\text { L'Hardy-Halos \& Rueness in Aponte } \text { et al. }\end{array}$ & $\mathrm{A}, \mathrm{P}$ & $\mathrm{AC}, \mathrm{RO}$ & $22,25,28$ \\
\hline
\end{tabular}

Subfamília Lithophylloideae

Amphiroa beauvoisii J.V. Lamour.

AC, BU, CF, MA, RO, SQ

$1,10,11,15,17,22,25,28,3$

\section{NEMALIALES}

Liagoraceae

$\mathrm{AC}, \mathrm{RO}$ 


\begin{tabular}{|c|c|c|c|}
\hline Táxons & $\begin{array}{l}\text { Distribuição } \\
\text { geográfica } \\
\text { mundial }\end{array}$ & Municípios & $\begin{array}{l}\text { Referência } \\
\text { bibliográfica }\end{array}$ \\
\hline Aglaothamnion cordatum (Boergesen) Feldm.-Maz. & \multicolumn{2}{|c|}{ A (Brasil), M, I AC } & 28,31 \\
\hline $\begin{array}{l}\text { Aglaothamnion felliponei (M. Howe) N. Aponte, } \\
\text { D.L. Ballant. \& J.N. Norris }\end{array}$ & A & AC, BU, CF, MA, SQ & $1,10,11,19,18,28,31$ \\
\hline Aglaothamnion halliae (Collins) N. Aponte, D.L. Ballant. \& J.N. Norris & A & RO & 34 \\
\hline $\begin{array}{l}\text { Aglaothamnion uruguayense (W.R. Taylor) N. Aponte, } \\
\text { D.L. Ballant. \& J.N. Norris }\end{array}$ & A & $\mathrm{AC}, \mathrm{BU}, \mathrm{CF}, \mathrm{RO}, \mathrm{SQ}$ & $1,10,11,25,28,3134$ \\
\hline Anotrichium tenue (C. Agardh) Nägeli & $\mathrm{A}, \mathrm{M}, \mathrm{I}$ & $\mathrm{AC}$ & $11,16,28,31$ \\
\hline Antithamnion antillanum Boergesen & $\mathrm{Tr}, \mathrm{Te}$ & $\mathrm{AC}$ & 28 \\
\hline Antithamnion villosum (Kütz.) Athanas. in Maggs \& Hommersand & $\mathrm{Te}$ & $\mathrm{AC}$ & 25,32 \\
\hline Antithamnionella atlantica (E.C. Oliveira) C.W. Schneid. & $\mathrm{Tr}, \mathrm{ST}$ & $\mathrm{AC}$ & 25 \\
\hline Antithamnionella boergesenii (Cormaci \& Furnari) Athanas. & $\mathrm{A}, \mathrm{M}$ & $\mathrm{AC}$ & 25,28 \\
\hline Callithamniella flexilis Baardseth & $\mathrm{Te}$ & $\mathrm{AC}$ & 25,28 \\
\hline Callithamniella tingitana (Schousb. ex Bornet) Feldm.-Maz. & $\mathrm{Te}, \mathrm{Tr}$ & $\mathrm{AC}$ & $1,25,34$ \\
\hline Callithamnion corymbosum (Sm.) Lyngb. & $\mathrm{Te}, \mathrm{Tr}$ & $\mathrm{BU}$ & 11 \\
\hline Callithamnion tetragonum (Withering) S.F. Gray & $\mathrm{Te}, \mathrm{Tr}$ & $\mathrm{AC}$ & 25 \\
\hline $\begin{array}{l}\text { Centroceras clavulatum (C. Agardh in Kunth) Mont. } \\
\text { in Durieu de Maisonneuve }\end{array}$ & $\mathrm{Tr}, \mathrm{Te}$ & AC, BU, CF, MA, SQ & $1,10,11,16,19,18,28,31$ \\
\hline Centrocerocolax ubatubensis A.B. Joly & A (Brasil) & AC, BU, CF, MA, SQ & $1,10,19,18,28$ \\
\hline Ceramium brasilense A.B. Joly & A (Brasil) & $\mathrm{AC}, \mathrm{BU}, \mathrm{CF}, \mathrm{MA}, \mathrm{SQ}$ & $1,3,10,11,16,19,18,28,31$ \\
\hline Ceramium brevizonatum H.E. Petersen & $\mathrm{A}, \mathrm{I}, \mathrm{P}$ & $\mathrm{BU}$ & 11 \\
\hline Ceramium brevizonatum var. caraibicum H.E. Petersen \& Boergesen & $\mathrm{A}, \mathrm{I}$ & $\mathrm{AC}, \mathrm{BU}$ & $3,4,22$ \\
\hline Ceramium codii (H. Richards) Maz. & $\mathrm{A}, \mathrm{I}, \mathrm{P}$ & $\mathrm{AC}$ & $10,28,31$ \\
\hline Ceramium comptum Boergesen & A, I, M & $\mathrm{AC}, \mathrm{BU}, \mathrm{RO}$ & $3,11,16,22,25$ \\
\hline Ceramium dawsonii A.B. Joly & $\mathrm{A}, \mathrm{I}$ & $\mathrm{AC}, \mathrm{BU}, \mathrm{MA}, \mathrm{RO}$ & $4,10,11,22$ \\
\hline Ceramium deslongchampsii Chauv. ex Duby & $\mathrm{C}$ & BU & 3 \\
\hline Ceramium diaphanum (Lightf.) Roth & $\mathrm{A}, \mathrm{I}$ & $\mathrm{AC}, \mathrm{MA}$ & $3,10,28,31$ \\
\hline Ceramium flaccidum (Kütz.) Ardiss. & $\mathrm{A}, \mathrm{I}$ & AC, BU, CF, MA, RO, SQ & $\begin{array}{l}1,3,4,10,11,16,17,19,18,22, \\
25,28,31\end{array}$ \\
\hline Ceramium luetzelburgii O.C. Schmidt & $\mathrm{A}, \mathrm{I}$ & $\mathrm{AC}, \mathrm{CF}$ & $3,10,28,31$ \\
\hline Ceramium tenerrimum (G. Martens) Okamura & $\mathrm{Tr}, \mathrm{Te}$ & AC, BU, CF, MA, SQ & $1,3,10,11,25,28,31$ \\
\hline Ceramium vagans P.C. Silva & $\operatorname{Tr}$ & $\mathrm{AC}, \mathrm{BU}, \mathrm{MA}$ & 1,3 \\
\hline
\end{tabular}




\begin{tabular}{|c|c|c|c|}
\hline Táxons & $\begin{array}{c}\text { Distribuição } \\
\text { geográfica } \\
\text { mundial }\end{array}$ & Municípios & $\begin{array}{l}\text { Referência } \\
\text { bibliográfica }\end{array}$ \\
\hline Corallophila apiculata (Yamada) R.E. Norris & A (Brasil), I, P & $\mathrm{AC}, \mathrm{BU}, \mathrm{CF}, \mathrm{SQ}$ & $1,3,28$ \\
\hline Crouania attenuata (C. Agardh) J. Agardh & $\mathrm{Tr}, \mathrm{Te}$ & $\mathrm{AC}$ & 25,28 \\
\hline Diplothamnion tetrastichum A.B. Joly \& Yamaguishi in Joly et al. & A & $\mathrm{RO}$ & 34 \\
\hline Dohrniella antillarum (W.R. Taylor) Feldm.-Maz. & A & BU & 11 \\
\hline Griffithsia schousboei Mont. & $\mathrm{A}, \mathrm{M}, \mathrm{P}$ & $\mathrm{AC}$ & $11,25,28,31$ \\
\hline Gymnothamnion elegans (Schousb. ex C. Agardh) J. Agardh & $\mathrm{A}, \mathrm{M}, \mathrm{P}$ & AC, BU, CF, MA, SQ & $1,10,16,19,18,28$ \\
\hline Pleonosporium polystichum E.C. Oliveira & $\mathrm{A}, \mathrm{I}, \mathrm{P}$ & $\mathrm{AC}, \mathrm{CF}$ & 25,28 \\
\hline Pterothamnion heteromorphum (J. Agardh) Athanasiadis \& Kraft & Te, Po & $\mathrm{RO}$ & 34 \\
\hline Ptilothamnion speluncarum (Collins \& Herv.) D.L. Ballant. \& M.J.Wynne & $\operatorname{Tr}$ & $\mathrm{AC}, \mathrm{BU}, \mathrm{CF}$ & 10,28 \\
\hline Spermothamnion nonatoi A.B. Joly & A (Brasil) & SQ & 1 \\
\hline Spyridia clavata Kütz. & $\mathrm{A}, \mathrm{MV}$ & $\mathrm{BU}$ & 11 \\
\hline Spyridia filamentosa (Wulfen) Harv. in Hook & $\mathrm{Tr}, \mathrm{Te}$ & $\mathrm{AC}, \mathrm{BU}, \mathrm{CF}$ & $10,11,16,19,20,18,22,28$ \\
\hline Spyridia hypnoides (Bory in Belanger) Papenf. & A & $\mathrm{AC}, \mathrm{BU}, \mathrm{CF}, \mathrm{MA}$ & $1,10,11,16,19,20,18,22,28,31$ \\
\hline Wrangelia argus (Mont.) Mont. & A, I, P & $\mathrm{AC}, \mathrm{BU}, \mathrm{CF}$ & $10,11,28,31$ \\
\hline \multicolumn{4}{|l|}{ Delesseriaceae } \\
\hline Acrosorium ciliolatum (Harv.) Kylin & $\mathrm{Tr}, \mathrm{Te}$ & $\mathrm{AC}, \mathrm{BU}, \mathrm{CF}, \mathrm{RO}$ & $11,22,25,28,3134$ \\
\hline Caloglossa leprieurii (Mont.) G. Martens & $\mathrm{A}, \mathrm{I}, \mathrm{P}$ & $\mathrm{BU}$ & 28 \\
\hline Cryptopleura ramosa (Hudson) Kylin ex L. Newton & A & $\mathrm{AC}, \mathrm{BU}, \mathrm{CF}, \mathrm{MA}, \mathrm{RO}$ & $1,19,18,22,25,28$ \\
\hline Gonimophyllum africanum M.T. Martin \& Pocock & $\mathrm{Te}$ & $\mathrm{AC}$ & 25,28 \\
\hline Haraldia tenuis E.C. Oliveira & A (Brasil) & $\mathrm{AC}$ & 28 \\
\hline Hypoglossum tenuifolium (Harv.) J. Agardh & $\mathrm{A}$ & $\mathrm{AC}$ & 25,28 \\
\hline Neuroglossum binderianum Kütz. & $\mathrm{A}, \mathrm{I}$ & $\mathrm{AC}$ & 28 \\
\hline Taenioma perpusillum (J. Agardh) J. Agardh & $\mathrm{Tr}, \mathrm{Te}$ & $\mathrm{CF}$ & 19,18 \\
\hline \multicolumn{4}{|l|}{ Sarcomeniaceae } \\
\hline Platysiphonia delicata (Clemente) Cremades & $\mathrm{A}, \mathrm{I}, \mathrm{P}$ & $\mathrm{AC}, \mathrm{BU}$ & 25,28 \\
\hline \multicolumn{4}{|l|}{ Dasyaceae } \\
\hline Dasya brasiliensis E.C. Oliveira \& Y. Braga & A & $\mathrm{AC}, \mathrm{BU}, \mathrm{CF}, \mathrm{MA}, \mathrm{RO}$ & $1,10,11,19,18,22,28,31$ \\
\hline Dasya corymbifera J. Agardh & $\mathrm{Te}, \mathrm{Tr}$ & $\mathrm{AC}, \mathrm{BU}, \mathrm{CF}$ & $10,11,19,18,22,28,31$ \\
\hline
\end{tabular}




\begin{tabular}{|c|c|c|c|}
\hline Táxons & $\begin{array}{l}\text { Distribuição } \\
\text { geográfica } \\
\text { mundial }\end{array}$ & Municípios & $\begin{array}{l}\text { Referência } \\
\text { bibliográfica }\end{array}$ \\
\hline Dasya elongata Sond. & A (Brasil), I & BU, RO & $22,28,31$ \\
\hline Dasya ocellata (Gratel.) Harv. in Hook. & $\mathrm{Tr}, \mathrm{Te}$ & $\mathrm{RO}$ & 34 \\
\hline Dasya rigidula (Kütz.) Ardiss. & A, I, M & $\mathrm{AC}, \mathrm{RO}$ & 25,34 \\
\hline Heterosiphonia crassipes (Harv.) Falkenb. & A (Brasil), I, P & BU & 28 \\
\hline Heterosiphonia crispella (C. Agardh) M.J. Wynne & $\mathrm{Tr}, \mathrm{Te}$ & $\mathrm{AC}, \mathrm{BU}, \mathrm{CF}$ & $10,16,19,18,25,28$ \\
\hline Heterosiphonia gibbesii (Harv.) Falkenb. & $\mathrm{A}, \mathrm{P}$ & $\mathrm{BU}$ & 10,11 \\
\hline \multicolumn{4}{|l|}{ Rhodomelaceae } \\
\hline Acanthophora muscoides (L.) Bory & $\mathrm{A}, \mathrm{I}, \mathrm{P}$ & $\mathrm{CF}$ & 11,28 \\
\hline Acanthophora spicifera (Vahl) Boergesen & $\mathrm{Tr}, \mathrm{Te}$ & $\mathrm{BU}, \mathrm{CF}, \mathrm{SP}$ & $10,11,19,20,18,22,28,31$ \\
\hline Bostrychia calliptera (Mont.) Mont. & $\mathrm{A}, \mathrm{I}, \mathrm{P}$ & $\mathrm{BU}$ & 28 \\
\hline Bostrychia montagnei Harv. & A & $\mathrm{BU}$ & 28 \\
\hline Bostrychia moritziana (Sond. ex Kütz.) J. Agardh & $\mathrm{A}, \mathrm{I}, \mathrm{P}$ & $\mathrm{CF}$ & 19,18 \\
\hline Bostrychia radicans (Mont.) Mont. in Orbigny & $\mathrm{A}, \mathrm{I}$ & BU, CF, MA, SQ & $1,10,19,18,28$ \\
\hline Bostrychia tenella (J.V. Lamour.) J. Agardh & $\mathrm{A}, \mathrm{I}, \mathrm{P}$ & $\mathrm{AC}, \mathrm{BU}, \mathrm{CF}, \mathrm{MA}, \mathrm{SQ}$ & $1,19,18,28$ \\
\hline Bryocladia cuspidata (J. Agardh) De Toni & A & CF, SQ & $1,11,28$ \\
\hline Bryocladia thyrsigera (J. Agardh) F. Schmitz in Falkenb. & A & $\mathrm{AC}, \mathrm{BU}, \mathrm{CF}, \mathrm{MA}, \mathrm{SQ}$ & $1,10,11,22,28$ \\
\hline Bryothamnion seaforthii (Turner) Kütz. & $\mathrm{A}, \mathrm{I}$ & $\mathrm{RO}$ & 22 \\
\hline Chondria atropurpurea Harv. & A & $\mathrm{BU}, \mathrm{CF}, \mathrm{SQ}$ & $1,11,28$ \\
\hline Chondria dasyphylla (Woodw.) C. Agardh & $\mathrm{C}$ & $\mathrm{AC}$ & 16 \\
\hline Chondria decipiens Kylin & $\mathrm{A}, \mathrm{I}$ & $\mathrm{BU}, \mathrm{CF}$ & 10,28 \\
\hline Chondria platyramea A.B. Joly \& Ugadim in Joly et al. & A & $\mathrm{AC}, \mathrm{BU}, \mathrm{MA}, \mathrm{RO}$ & $1,16,22$ \\
\hline Chondria polyrhiza Collins \& Herv. & $\mathrm{A}, \mathrm{I}, \mathrm{P}$ & $\mathrm{AC}, \mathrm{BU}, \mathrm{CF}, \mathrm{MA}, \mathrm{SQ}$ & $1,10,11,19,18,22,28$ \\
\hline Chondrophycus corallopsis (Mont.) K.W. Nam & $\mathrm{A}, \mathrm{I}$ & BU & 11 \\
\hline Chondrophycus flagelliferus (J. Agardh) K.W. Nam & $A, I$ & $\mathrm{BU}, \mathrm{CF}$ & $10,11,22,28$ \\
\hline Chondrophycus papillosus (C. Agardh) Garbary \& J.T. Harper & $\mathrm{Te}, \mathrm{Tr}$ & BU & 11 \\
\hline Chondrophycus translucidus (Fujii \& Cord.-Mar.) Garbary \& J.T. Harper & A (Brasil) & BU & 11 \\
\hline $\begin{array}{l}\text { Dawsoniocolax bostrychiae (A.B. Joly \& Yam.-Tomita) } \\
\text { A.B. Joly \& Yam.-Tomita }\end{array}$ & A (Brasil), $\mathrm{P}$ & $\mathrm{BU}$ & 28 \\
\hline Herposiphonia bipinnata M. Howe & A & $\mathrm{AC}, \mathrm{BU}, \mathrm{CF}$ & $10,11,25,28,31$ \\
\hline Herposiphonia secunda (C. Agardh) Ambronn & $\mathrm{Tr}, \mathrm{Te}$ & $\mathrm{AC}, \mathrm{BU}, \mathrm{CF}, \mathrm{MA}, \mathrm{RO}, \mathrm{SQ}$ & $1,10,11,19,18,22$ \\
\hline Herposiphonia tenella (C. Agardh) Ambronn & $\mathrm{Tr}, \mathrm{Te}$ & $\mathrm{AC}, \mathrm{BU}, \mathrm{CF}, \mathrm{MA}, \mathrm{RO}, \mathrm{SQ}$ & $1,10,19,18,22,25,28,31$ \\
\hline
\end{tabular}




\begin{tabular}{|c|c|c|c|}
\hline Táxons & $\begin{array}{c}\text { Distribuição } \\
\text { geográfica } \\
\text { mundial }\end{array}$ & Municípios & $\begin{array}{l}\text { Referência } \\
\text { bibliográfica }\end{array}$ \\
\hline Laurencia intricata J.V. Lamour. & $\mathrm{Te}, \mathrm{Tr}$ & $\mathrm{BU}$ & 11 \\
\hline Laurencia obtusa (Huds.) J.V. Lamour. & A (Brasil), P & $\mathrm{AC}, \mathrm{BU}, \mathrm{CF}$, & $10,19,18,28,31$ \\
\hline Laurencia oliveirana Yonesh. & A (Brasil) & $\mathrm{AC}$ & $11,28,31$ \\
\hline Lophosiphonia cristata Falkenb. & $\mathrm{A}, \mathrm{M}, \mathrm{P}$ & $\mathrm{AC}$ & $10,28,31,30$ \\
\hline Murrayella periclados (C. Agardh) F. Schmitz & $\mathrm{A}, \mathrm{P}$ & $\mathrm{AC}, \mathrm{BU}, \mathrm{CF}$ & $19,18,28$ \\
\hline Neosiphonia ferulacea (Suhr ex J. Agardh) S.M. Guim. \& M.T. Fujii & $\mathrm{A}, \mathrm{I}, \mathrm{P}$ & $\mathrm{AC}, \mathrm{BU}, \mathrm{CF}, \mathrm{MA}, \mathrm{SQ}$ & $1,10,16,28,31$ \\
\hline Neosiphonia flaccidissima (Hollenb.) M.-S. Kim \& I.K. Lee & $\mathrm{A}, \mathrm{P}$ & $\mathrm{AC}, \mathrm{CF}$ & $25,28,29,31$ \\
\hline Neosiphonia sphaerocarpa (Boergesen) M.-S. Kim \& I.K. Lee & A, I, P & $\mathrm{AC}, \mathrm{BU}$ & $10,28,29$ \\
\hline Neosiphonia tongatensis (Harv. ex Kütz.) M.-S. Kim \& I. K. Lee & $\mathrm{C}$ & $\mathrm{AC}, \mathrm{BU}, \mathrm{CF}, \mathrm{SP}$ & $17,19,18,28,29,31$ \\
\hline Ophidocladus simpliciusculus (P. Crouan \& H. Crouan) Falkenb. & $\mathrm{A}, \mathrm{I}$ & $\mathrm{AC}, \mathrm{BU}, \mathrm{CF}, \mathrm{MA}, \mathrm{SQ}$ & $1,10,11,19,18,28$ \\
\hline Osmundaria obtusiloba (C. Agardh) R.E. Norris & A, P & BU, CF, SQ & $1,10,28$ \\
\hline Osmundea lata (M. Howe \& W.R. Taylor) Yonesh., M.T. Fujii \& Gurgel & A (Brasil) & $\mathrm{CF}$ & 33 \\
\hline Polysiphonia decussata Hollenb. & A (Brasil), I, P & $\mathrm{AC}, \mathrm{CF}, \mathrm{MA}$ & $10,19,18,25,28,31$ \\
\hline Polysiphonia denudata (Dillwyn) Grev. ex Harv. in Hook. & $\mathrm{A}, \mathrm{I}, \mathrm{P}$ & $\mathrm{AC}$ & $10,16,28$ \\
\hline Polysiphonia howei Hollenb. in W.R. Taylor & $\mathrm{A}, \mathrm{I}, \mathrm{P}$ & AC, BU, MA, RO, SQ & $1,10,22,28$ \\
\hline Polysiphonia saccorhiza(Collins \& Herv.) Hollenb. & $\mathrm{A}, \mathrm{M}, \mathrm{P}$ & $\mathrm{AC}, \mathrm{BU}$ & 10,28 \\
\hline Polysiphonia scopulorum Harv. & $\mathrm{A}, \mathrm{I}, \mathrm{P}$ & $\mathrm{AC}, \mathrm{BU}, \mathrm{SQ}$ & $1,10,11,28$ \\
\hline Polysiphonia scopulorum var. villum (J. Agardh) Hollenb. & $\mathrm{C}$ & $\mathrm{AC}, \mathrm{BU}$ & $25,28,31$ \\
\hline Polysiphonia sertularioides (Grateloup) J. Agardh & $\mathrm{Te}, \mathrm{Tr}$ & $\mathrm{AC}, \mathrm{BU}$ & 10 \\
\hline Polysiphonia subtilissima Mont. & $\mathrm{A}, \mathrm{I}, \mathrm{P}$ & $\mathrm{AC}, \mathrm{CF}$ & $11,19,18,25$ \\
\hline Pterosiphonia parasitica (Hudson) Falkenberg & $\mathrm{Te}, \mathrm{Tr}$ & $\mathrm{AC}, \mathrm{BU}, \mathrm{CF}$ & $1,10,25,28,31$ \\
\hline Pterosiphonia parasítica var. australis A.B. Joly \& Cord.-Mar. & A (Brasil) & $\mathrm{BU}, \mathrm{CF}$ & 31 \\
\hline Pterosiphonia pennata (C. Agardh) Falkenb. & A (Brasil) & $\mathrm{AC}, \mathrm{BU}, \mathrm{MA}, \mathrm{RO}, \mathrm{SQ}$ & $1,10,11,16,22,28,34$ \\
\hline Pterosiphonia spinifera (Kütz.) Ardré & $\mathrm{Te}$ & AC, BU, MA, SQ & $1,10,25,28,29$ \\
\hline Streblocladia corymbifera (C. Agardh) Kylin & A (Brasil), I, P & $\mathrm{AC}$ & $25,28,29,31$ \\
\hline Wrightiella tumanowiczii (Gatty ex Harv.) F. Schmitz & A & $\mathrm{RO}$ & 34 \\
\hline \multicolumn{4}{|l|}{ GELIDIALES } \\
\hline Gelidium crinale (Turner) Gaillon & $\mathrm{A}, \mathrm{I}, \mathrm{P}$ & $\mathrm{AC}, \mathrm{CF}, \mathrm{SQ}$ & $1,28,31$ \\
\hline Gelidium floridanum W.R. Taylor & $\mathrm{A}$ & $\mathrm{AC}, \mathrm{MA}$ & 1,25 \\
\hline Gelidium pusillum (Stackh.) Le Jolis & $\mathrm{C}$ & $\mathrm{AC}, \mathrm{BU}, \mathrm{CF}, \mathrm{MA}, \mathrm{SQ}$ & $1,10,19,18,25,28,31$ \\
\hline
\end{tabular}




\begin{tabular}{|c|c|c|c|}
\hline Táxons & $\begin{array}{c}\text { Distribuição } \\
\text { geográfica } \\
\text { mundial }\end{array}$ & Municípios & $\begin{array}{l}\text { Referência } \\
\text { bibliográfica }\end{array}$ \\
\hline Gelidium spinosum (S.G Gmel.) P.C. Silva & $\mathrm{Te}, \mathrm{Tr}$ & $\mathrm{AC}, \mathrm{BU}, \mathrm{SQ}$ & $1,25,28$ \\
\hline Pterocladiella capillacea (S.G. Gmel.) Santel \& Hommers. & $\mathrm{Te}$ & $\mathrm{AC}, \mathrm{BU}, \mathrm{CF}, \mathrm{MA}, \mathrm{RO}, \mathrm{SQ}$ & $1,10,16,19,18,22,25,28,31,34$ \\
\hline \multicolumn{4}{|l|}{ Gelidiellaceae } \\
\hline Gelidiella acerosa (Forssk.) Feldmann \& Hamel & $\mathrm{Tr}, \mathrm{Te}$ & $\mathrm{BU}$ & 10,28 \\
\hline Gelidiella trinitatensis W.R. Taylor & A & SQ & 1,11 \\
\hline Parviphycus tenuissimus (Feldmann \& Hamel) Santel. & $\mathrm{Te}, \mathrm{Tr}$ & $\mathrm{AC}$ & 28,31 \\
\hline \multicolumn{4}{|l|}{ GIGARTINALES } \\
\hline Calliblepharis fimbriata (Grev.) Kütz. & A & $\mathrm{BU}$ & 22,28 \\
\hline Hypnea cenomyce J. Agardh & $\mathrm{A}, \mathrm{I}, \mathrm{P}$ & $\mathrm{AC}, \mathrm{SQ}$ & 1,28 \\
\hline Hypnea musciformis (Wulfen in Jacquin) J.V. Lamour. & $\mathrm{Tr}, \mathrm{Te}$ & $\mathrm{AC}, \mathrm{BU}, \mathrm{CF}, \mathrm{MA}, \mathrm{RO}, \mathrm{SQ}$ & $1,6,10,11,19,20,18,22,25,28,31$ \\
\hline Hypnea spinella (C. Agardh) Kütz. & $\mathrm{A}, \mathrm{P}$ & AC, BU, CF, MA, SQ & $\begin{array}{l}1,10,11,16,17,19,20,18,22 \\
28,31\end{array}$ \\
\hline Hypnea valentiae (Turner) Mont. & $\mathrm{A}, \mathrm{I}, \mathrm{P}$ & $\mathrm{BU}, \mathrm{CF}, \mathrm{SP}$ & $19,18,28$ \\
\hline Hypneocolax stellaris Boergesen & $\mathrm{A}, \mathrm{I}$ & SQ & 1 \\
\hline \multicolumn{4}{|l|}{ Gigartinaceae } \\
\hline Chondracanthus acicularis (Roth) Fredericq & $\mathrm{Tr}, \mathrm{Te}$ & $\mathrm{AC}, \mathrm{BU}, \mathrm{CF}, \mathrm{MA}, \mathrm{SQ}$ & $1,10,11,19,18,28,31$ \\
\hline Chondracanthus teedei (Mertens ex Roth) Fredericq & $\mathrm{A}, \mathrm{I}, \mathrm{P}$ & AC, BU, CF, MA, SQ & $1,10,11,16,19,18,22,28,31$ \\
\hline \multicolumn{4}{|l|}{ Kallymeniaceae } \\
\hline Callophyllis microdonta (Grev.) Falkenb. & $\mathrm{A}, \mathrm{I}$ & $\mathrm{AC}$ & 25,28 \\
\hline \multicolumn{4}{|l|}{ Peyssonneliaceae } \\
\hline Peyssonnelia boudouresquei Yonesh. & $\mathrm{A}, \mathrm{I}$ & $\mathrm{AC}, \mathrm{SQ}$ & $1,25,26,28,31$ \\
\hline Peyssonnelia capensis Mont. & $\mathrm{A}, \mathrm{I}, \mathrm{P}$ & $\mathrm{AC}, \mathrm{CF}$ & 25,28 \\
\hline Peyssonnelia inamoema Pilg. & $\mathrm{A}, \mathrm{M}, \mathrm{P}$ & $\mathrm{BU}$ & 28 \\
\hline Peyssonnelia valentinii Yonesh. \& Boudour. & A & $\mathrm{AC}$ & 25,28 \\
\hline
\end{tabular}




\begin{tabular}{|c|c|c|c|}
\hline Táxons & $\begin{array}{c}\text { Distribuição } \\
\text { geográfica } \\
\text { mundial }\end{array}$ & Municípios & $\begin{array}{c}\text { Referência } \\
\text { bibliográfica }\end{array}$ \\
\hline \multicolumn{4}{|l|}{ Solieriaceae } \\
\hline Wurdemannia miniata (Spreng.) Feldmann \& Hamel & $\mathrm{Tr}, \mathrm{Te}$ & $\mathrm{AC}, \mathrm{BU}, \mathrm{CF}$ & 28,31 \\
\hline \multicolumn{4}{|l|}{ Phyllophoraceae } \\
\hline Gymnogongrus griffithsiae (Turner) Mart. & $\mathrm{A}, \mathrm{M}$ & $\mathrm{AC}, \mathrm{BU}, \mathrm{CF}, \mathrm{MA}, \mathrm{SQ}$ & $1,10,16,19,18,28,31$ \\
\hline Petroglossum undulatum C.W. Schneid. in C.W. Schneid. \& Searles & A & $\mathrm{RO}$ & 34 \\
\hline \multicolumn{4}{|l|}{ GRACILARIALES } \\
\hline Gracilaria brasiliensis Gurgel \& Yonesh. & A (Brasil) & BU & 13 \\
\hline Gracilaria blodgettii Harv. & $\mathrm{Te}, \mathrm{Tr}$ & $\mathrm{BU}$ & 28 \\
\hline Gracilaria cervicornis (Turner) J. Agardh & $\mathrm{A}, \mathrm{I}, \mathrm{M}$ & $\mathrm{AC}, \mathrm{BU}, \mathrm{CF}, \mathrm{SP}, \mathrm{SQ}$ & $1,10,19,20,18,28$ \\
\hline Gracilaria domingensis (Kütz.) Sond. ex Dickie & $\mathrm{A}$ & $\mathrm{BU}$ & 28 \\
\hline Gracilaria mammillaris (Mont.) M. Howe & $\mathrm{A}, \mathrm{P}$ & MA & 1 \\
\hline Gracilaria tepocensis (E.Y. Dawson) E.Y. Dawson & $\mathrm{A}, \mathrm{P}$ & $\mathrm{BU}$ & 28 \\
\hline Gracilaria yoneshigueana Gurgel, Fredericq \& J. Norris & A (Brasil) & $\mathrm{BU}$ & 12 \\
\hline \multicolumn{4}{|l|}{ Pterocladiophilaceae } \\
\hline Gelidiocolax pustulata E.C. Oliveira \& Yonesh. & A (Brasil) & $\mathrm{AC}, \mathrm{SQ}$ & $1,25,27,28,31$ \\
\hline \multicolumn{4}{|l|}{ HALYMENIALES } \\
\hline \multicolumn{4}{|l|}{ Halymeniaceae } \\
\hline Cryptonemia crenulata (J. Agardh) J. Agardh & $\mathrm{A}, \mathrm{I}, \mathrm{P}$ & $\mathrm{RO}$ & 34 \\
\hline Cryptonemia delicatula A.B. Joly \& Cordeiro in Joly et al. & A & $\mathrm{RO}$ & 34 \\
\hline Cryptonemia flabellifolia Pinheiro-Joventino \& E.C. Oliveira & A (Brasil) & $\mathrm{RO}$ & 34 \\
\hline Cryptonemia limensis (Kütz.) J.A. Lewis & $\mathrm{A}, \mathrm{P}$ & $\mathrm{AC}$ & 28 \\
\hline Cryptonemia seminervis (C. Agardh) J. Agardh & $\mathrm{A}, \mathrm{I}, \mathrm{P}$ & AC, BU, CF, MA, RO, SQ & $1,22,25,28,34$ \\
\hline Grateloupia turuturu Yamada & $\mathrm{A}, \mathrm{M}, \mathrm{P}$ & $\mathrm{RO}$ & 22Széchy (1996) \\
\hline Grateloupia filicina (J.V. Lamour.) C. Agardh & $\mathrm{C}$ & AC, BU, CF, MA, RO, SQ & $1,10,11,22,28$ \\
\hline Halymenia floridana J. Agardh & $\mathrm{A}, \mathrm{I}$ & BU, RO & 28,34 \\
\hline
\end{tabular}

\section{GRACILARIALES}

Gracilariaceae

Gracilaria blodgettii Harv.

$\mathrm{BU}$

A, I,

Gracilaria mammillaris (Mont.) M. Howe

$\mathrm{A}, \mathrm{P}$

MA

A (Bra

Pterocladiophilaceae 


\section{Táxons}

Distribuição Municípios

Referência

\section{PLOCAMIALES}

\section{Plocamiacea}

Plocamium brasiliense (Grev. in J. St-Hil.) M. Howe \& W.R. Taylor

\section{RHODYMENIALES}

\section{Rhodymeniaceae}

Asteromenia peltata (W.R. Taylor) Huisman \& A. Millar

Botryocladia occidentalis (Boergesen) Kylin

Botryocladia pyriformis (Boergesen) Kylin

Rhodymenia pseudopalmata (J.V. Lamour.) P.C. Silva

$\begin{array}{lll}\text { A, I, P } & \text { RO } & 34 \\ \text { A } & \text { RO } & 24,34 \\ \text { A, I } & \text { RO } & 34 \\ \text { Tr, Te } & \text { AC, BU, CF, MA, SQ } & 1,19,18,22,28\end{array}$

\section{Faucheaceae}

Gloiocladia iyoensis (Okamura) R.E. Norris

Leptofauchea brasiliensis A.B. Joly

A (Brasil), I, P AC

A

BU, MA

Champia feldmannii Diaz-Pif.

Champia parvula (C. Agardh) Harv.

Champia vieillardii Kütz.

Gastroclonium parvum (Hollenb.) C.F. Chang \& B.M. Xia

\section{Lomentariaceae}

Gelidiopsis planicaulis (W.R. Taylor) W.R. Taylor

Gelidiopsis variabilis (Grev. ex J. Agardh) F. Schmitz

Lomentaria corallicola Boergesen

Lomentaria rawitscheri A. B. Joly
$\mathrm{Tr}, \mathrm{Te}$

Te $\quad A C$

A, I, P AC, BU, CF, MA, RO, SQ

A (Brasil), $\mathrm{P} \quad \mathrm{AC}, \mathrm{BU}, \mathrm{CF}, \mathrm{MA}, \mathrm{SQ}$

$\begin{array}{lll}\text { A } & \text { AC } & 11,28,31 \\ \text { A, I, P } & \text { AC, BU } & 10,11,16,22,28,31 \\ \text { A (Brasil), I, P } & \text { AC } & 28\end{array}$

A (Brasil), I, P AC

A (Brasil) AC, MA, SQ
$1,11,17,22,25,28,31$

$1,10,19,18,28,31$

$1,25,28,31$ 


\section{REFERÊNCIAS BibLIOGRÁfICAS}

André, D. L.; Oliveira, M. C.; Okuda, T.; Horta, A. M. T. C.; Soldan, A. L.; Moreira, I. M. N. S.; Rollemberg, M. C. E. \& Heinzen, V. E. F. 1981. Estudo preliminar sobre as condições hidroquímicas da Lagoa de Araruama - Rio de Janeiro. Instituto de Pesquisas da Marinha 139: 1-14.

Allard, P. 1955. Anomalies dans les temperatures de léuax de mer observée au Cabo Frio (Brésil). Bulletin d'Information. Comite Central d'Oceanographie d'Etude des Cotes 2: 58-63.

Amado Filho, G. M. 1991. Algas marinhas bentônicas do litoral de Saquarema a Itacoatiara (RJ). Dissertação de Mestrado. Universidade Federal do Rio de Janeiro, Rio de Janeiro, 322p.

\& Bahia, R. G. 2008. Algas marinhas bentônicas do estado do Rio de Janeiro. http://www.jbrj.gov.br/jabot/mapa/ algasrj.php. Acessado em 12 de agosto de 2008.

\& Yoneshigue-Valentin, Y. 1990/92. Feofíceas novas e raras para o litoral brasileiro. Rodriguésia 42/44: 39-46.

Barbiére, E. B. 1985. Condições climáticas dominantes na porção oriental da Lagoa de Araruama (RJ) e suas implicações na diversidade e teor de salinidade. Caderno de Ciências da Terra 59: 3-35.

Barreto, M. B. B. B. 1996. Aspectos morfológicos do gênero Ceramium Roth (Ceramiaceae, Rhodophyta) no estado do Rio de Janeiro. Dissertação de Mestrado. Universidade Federal do Rio de Janeiro, Rio de Janeiro, 134p.

Barros-Barreto, M. B.; McIvor, L.; Maggs, C. A. \& Ferreira, P. C. G. 2006. Molecular systematics of Ceramium and Centroceras (Ceramiaceae, Rhodophyta) from Brazil. Journal of Phycology 42: 905-921.

Bravin, I. C.; Torres, J.; Gurgel, C. F. D. \& Yoneshigue-Valentin, Y. 1999. Novas ocorrências de clorofíceas marinhas de profundidade para o Brasil. Hoehnea 26(2): 121-133.
\& Yoneshigue-Valentin, Y. 2002. Influência de fatores ambientais sobre o crescimento in vitro de Hypnea musciformis (Wulfen) Lamouroux (Rhodophyta). Revista Brasileira de Botânica 25(4): 469-474.

Cassano, V. 1997. Taxonomia e morfologia de Ectocarpus breviarticulatus, Feldmannia indica, Feldmannia irregularis, Hincksia conifera e Hincksia mitchelliae (Ectocarpaceae, Phaeophyta) no estado do Rio de Janeiro. Universidade Federal do Rio de Janeiro, Rio de Janeiro, 211p.

Yoneshigue-Valentin, Y. \& Wynne, M. J. 2004. Elachistiella leptonematoides gen. et. sp. nov. (Elachistaceae, Phaeophyceae) from Brazil. Phycologia 43(3): 329-340.

CILSJ. 2008. Consórcio Intermunicipal para gestão ambiental das bacias da Região dos Lagos, do Rio São João e Zona Costeira. Disponível em http://www.lagossaojoao. org.br/index-cilsj.html. Acessado em: 22 de maio de 2008.

Emilson, I. 1961. The shelf and coastal waters of southern Brazil. Boletim do Instituto Oceanográfico 2: 101-112.

Braga, M. R. A.; Cordeiro-Marino, M. \& Pedrini, A. G. 1986. Morphology and taxonomy of Jolyna laminarioides, a new member of the Scytosiphonales (Phaeophyceae) from Brazil. Phycologia (1): 99-108.

Guimarães, M. A. \& Coutinho, R. 1996. Spatial and temporal variation of benthic marine algae at the Cabo Frio upwelling region, Rio de Janeiro, Brazil. Aquatic Botany 52: 283-299.

Guiry, M. D. \& Guiry, G. M. 2008. AlgaeBase. World-wide electronic publication, National University of Ireland, Galway. http://www.algaebase.org. Acessado em 12 de agosto de 2008.

Gurgel, C. F. D. 1997. Estudo qualitativo e quantitativo das populações de macroalgas de uma comunidade bentônica sob impacto antropogênico. Dissertação de Mestrado. 
Universidade Federal do Rio de Janeiro, Rio de Janeiro, 65p.

Gurgel, C. F. D.; Fredericq, S. \& Norris, J. N. 2004. Molecular systematics and taxonomy of flattened species of Gracilaria Greville (Gracilariaceae, Gracilariales, Rhodophyta) from the western Atlantic. In: Abbott, I.A. and McDermid, K.J. (eds.). Taxonomy of economic seaweeds, with reference to the Pacific and other locations. University of Hawaii, Honolulu. Pp. 159-199.

Gurgel, C. F. D.; Fredericq, S.; Norris, J. N. \& Yoneshigue-Valentin, Y. 2008. Two new flat species of Gracilaria (Gracilariales, Rhodophyta) from Brazil: G. abyssalis sp. nov. and $G$. brasiliensis sp. nov. Phycologia 47(3): 249-264.

Horta, P. A.; Amancio, E.; Coimbra. C. S. \& Oliveira, E. C. 2001. Considerações sobre a distribuição e origem da flora de macroalgas marinhas brasileiras. Hoehnea 28(3): 243-265.

Mascarenhas, A. S. Jr.; Miranda, L. M. \& Rock, N. J. 1971. A study of the oceanographic conditions in the region of Cabo Frio. In: Costlow, J. D. (ed.). Fertility of the sea. Gordon \& Breach Scientific Publication, New York. Pp. 285-295.

Mitchell, G. J. P.; Széchy, M. T. M. \& Mitsuya, L. A. 1979. Sinópse das clorofíceas marinhas bentônicas do litoral do estado do Rio de Janeiro. Leandra 8-9: 91-123.

Moreira da Silva, P. C. 1968. O fenômeno da ressurgência na costa meridional brasileira. Instituto de Pesquisas da Marinha 24: 1-38. 1971. Upwelling and its biological effects in Southern Brazil. In: Costlow, J. D. (ed.). Fertility of the Sea. New York, Gordon \& Breach Scientif Publication. New York. Pp. 469-474.

Moura, C. W. N. 2000. Coralináceas com genículo (Rhodophyta, Corallinales) do litoral brasileiro. Tese de Doutorado. Universidade de São Paulo, 264p.

Muniz, R. A.; Gonçalves, J. E. A. \& Széchy, M. T. M. 2003. Variação temporal das macroalgas epífitas em Sargassum vulgare C. Agardh (Phaeophyta, Fucales) da Prainha, Arraial do Cabo, Rio de Janeiro, Brasil. Iheringia 58(1): 13-24.

Oigman-Pszczol, S. S.; Figueiredo, M. A. O. \& Creed, J. C. 2004. Distribution of benthic communities on the tropical rocky subtidal of Armação dos Búzios, southeastern Brazil. Marine Ecology 25 (3): 173-190.

Oliveira, E. C., Horta, P. A., Amancio, C. E. \& Silva, B. N. T. 2008. Algae Maris Brasilis. http://www.ib.usp.br/algaemaris/ algaemarisbrasilis.html. Acessado em 14 de agosto de 2008.

Oliveira Filho, E. C. 1977. Algas Marinhas Bentônicas do Brasil. Tese de Livre Docência. Universidade de São Paulo, São Paulo, 407p.

Palacios, J. R. 1993. Estudo espectral do fenômeno da ressurgência de Cabo Frio (RJ, Brasil). Dissertação de Mestrado. CNPq, Rio de Janeiro, 108 p.

Reis, R. P. \& Yoneshigue-Valentin, Y. 1996. Distribuição das macroalgas na Lagoa de Araruama, estado do Rio de Janeiro, Brasil. Revista Brasileira de Botânica 19(1): 77-85. \& Yoneshigue-Valentin, Y. 1998. Variação espaço-temporal de populações de Hypnea musciformis (Rhodophyta, Gigartinales) na baía de Sepetiba e Armação dos Búzios, RJ, Brasil. Acta Botanica Brasilica 12(3): 465-483.

Reis-Santos, R. P. 1990. Flora algal da Lagoa de Araruama, Rio de Janeiro. Dissertação de Mestrado. Universidade Federal do Rio de Janeiro, Rio de Janeiro, 319p.

Rodrigues, R. F. 1973. Upwelling at Cabo Frio (Brazil). Master Thesis. Naval Postgraduate School, Monterrey, 89p.

Signorini, S. R. 1978. On the circulation and the volume transport of the Brazil current between the Cape of São Tomé and Guanabara Bay. Deep Sea Research 25(5): 453-443.

Silva, G. L.; Dourado, M. S. \& Candella, R. N. 2006. Estudo preliminar da climatologia da ressurgência da região de Arraial do 
Cabo, RJ. Anais do XI Encontro Nacional dos Grupos PET, 16 a 21 de julho, Universidade de Santa Catarina, Florianópolis, SC, 11p.

Széchy, M. T. M. 1996. Estrutura de bancos de Sargassum (Phaeophyta - Fucales) do litoral dos estados do Rio de Janeiro e São Paulo. Tese de Doutorado. Universidade de São Paulo, 345p.

\& Cordeiro-Marino, M. 1991. Feofíceas do litoral norte do estado do Rio de Janeiro. Hoehnea 18: 205-241.

Tâmega, F. T. S \& Figueiredo, M. A. O. 2005. Distribuição das algas calcárias incrustantes (Corallinales, Rhodophyta) em diferentes habitats na Praia do Forno, Armação dos Búzios, Rio de Janeiro. Rodriguésia 56(87): 123-132.

Teixeira, V. L.; Pereira, R. C.; Muniz, J. A. \& Silva, L. F. F. 1985. Contribuição ao estudo de algas de profundidade da costa sudeste do Brasil. Ciência e Cultura 37(5): 809-815.

Torres Jr., A. R. 1995. Resposta da ressurgência costeira de Cabo Frio a forçantes locais. Dissertação de Mestrado. Universidade Federal do Rio de Janeiro, Riode Janeiro, 143p.

Valentin, J. L. 1974. O planctôn na ressurgência de Cabo Frio (Brasil) II. Primeiras observações sobre a estrutura física, química e biológica das águas da estação fixa (período 04/02 à 16/04/1973). Instituto de Pesquisas da Marinha 83: 1-11.

1983. L'écologie du plancton dans la remontée de Cabo Frio (Brésil). Thèse Docteur d'État-Sciences. Université d'Aix Marseille II, 254p.

1984. Analyse dês paramètres hidrobiologiques dans remontée de Cabo Frio, Brésil. Marine Biology 82: 259-276.

Valentin, J. L.; André, D. L. \& Jacob, S. A. 1987. Hydrobiology in the Cabo Frio (Brazil) upwelling: two dimensional structure and variability during a wind cycle. Continental Shelf Research 7(1): 77-88.

Villaça, R. C. 1988. Le phytobenthos des biotopes sciaphiles dans la region d'uppwelling de
Cabo Frio (Bresil). Thèse Docteur d'ÉtatSciences. Universite d'Aix de Marseille, $219 \mathrm{p}$.

Wynne, M. J. 2005. A checklist of benthic marine algae of tropical and subtropical western Atlantic: first revision. Nova Hedwigia 129: 1-152.

Yoneshigue, Y. 1985. Taxonomie et ecologie des algues marines dans la region de Cabo Frio (Rio de Janeiro, Bresil). Thèse Docteur d'État-Sciences. Université d'Aix Marseille II, 466p.

; Boudouresque, C. F. \& Figueireido, M. A. O. 1986. Flore marine de la région de Cabo Frio, État de Rio de Janeiro (Brésil). 5 - Sur Boodlea composita (Boodleaceae-Chlorophyta), Dictyota pardalis (Dictyotaceae-Phaeophyta) et Lophosiphonia cristata (RhodomelaceaeRhodophyta). Espèces nouvelles pour la cote brésilienne. Rickia 13: 17-27.

\& Figueireido, M. A. O. 1983. Flore marine de la région de Cabo Frio (Brésil) 3. Ectocarpaceae (Phaeophyta) nouvelles pour la cote brésilienne. Vie Milieu 33(3/ 4): 181-190.

\& Oliveira Filho, E. C. 1984. Algae from Cabo Frio upwelling área. 2. Gelidiocolax pustulata (Gelidiaceae, Rhodophyta): an usual new putative parasitic species. Journal of Phycology 20: 440-443. \& Valentin J. L. 1988. Comunidades algais fotófilas do infralitoral de Cabo Frio, Rio de Janeiro, Brasil. Gayana 45(1/4): 61-75. \& Villaça, R. C. 1986. Flora marinha da região de Cabo Frio (estado do Rio de Janeiro, Brasil). 6.Pterosiphonia spinifera, Polysiphonia eastwoodae, P. flaccidissima, P. sphaerocarpa e Streblocladia corymbifera (Rhodomelaceae, Rhodophyta). Novas ocorrências para a costa brasileira. Rickia 13: 97-111.

\& Villaça, R. C. 1989. Antithamnion tenuissimum (Ceramiaceae, Rhodophyta) dans la région de Cabo Frio (État de Rio de Janeiro, Brésil). Première citation pour 
l'Atlantique Sud. Cryptogamie Algologie 10(1): 325-335.

Yoneshigue-Valentin, Y.; Fujii, M. T. \& Gurgel, C. F. D. 2003. Osmundea lata (M. Howe \& W.R. Taylor) comb. nov. (Ceramiales, Rhodophyta) from the Brazilian south-eastern continental shelf. Phycologia 42(3): 301-307.

; Gestinari, L. M. S. \& Fernandes, D. R. P. 2006. Capítulo 2. Macroalgas. In: Lavrado, H. P. \& Ignacio, B. L. (eds.).
Biodiversidade bentônica da região central da Zona Econômica Exclusiva Brasileira. Série Livros n. 18. Museu Nacional, Rio de Janeiro. Pp. 67-105.

\& Valentin, J. L. 1992. Macroalgae of the Cabo Frio. Upwelling region, Brazil: ordination of communities. In: Seeliger, U. (ed.). Coastal plant communities of Latin America. Academic Press, San Diego. Pp. 31-50. 